\title{
Contribution to the knowledge of the macroinvertebrate fauna of the streams of Kahuzi- Biega National Park, Democratic Republic of Congo
}

MF Ngera, SU Pauls, RW Holzenthal, M Bagalwa, MA Bisimwa, EM Mushayuma \& DR Cammaerts

To cite this article: MF Ngera, SU Pauls, RW Holzenthal, M Bagalwa, MA Bisimwa, EM Mushayuma \& DR Cammaerts (2019) Contribution to the knowledge of the macroinvertebrate fauna of the streams of Kahuzi-Biega National Park, Democratic Republic of Congo, African Journal of Aquatic Science, 44:2, 127-142, DOI: 10.2989/16085914.2019.1598840

To link to this article: https://doi.org/10.2989/16085914.2019.1598840

曲 Published online: 31 Jul 2019.

Submit your article to this journal $\pi$

山 Article views: 23

Q View related articles $\sqsubset$

View Crossmark data $\nearrow$ 


\title{
Contribution to the knowledge of the macroinvertebrate fauna of the streams of Kahuzi-Biega National Park, Democratic Republic of Congo
}

\author{
MF Ngera ${ }^{1 * \ddagger}$, SU Pauls ${ }^{2}$, RW Holzenthal ${ }^{3}$, M Bagalwa $^{1}$, MA Bisimwa ${ }^{1}$, EM Mushayuma ${ }^{1}$ and DR Cammaerts ${ }^{4}$ \\ ${ }^{1}$ Centre de Recherche en Sciences Naturelles Lwiro, Bukavu, Democratic Republic of Congo \\ 2 Senckenberg Research Institute and Natural History Museum, Frankfurt am Main, Germany \\ ${ }^{3}$ Department of Entomology, University of Minnesota, St Paul, MN, USA \\ ${ }^{4}$ Independent researcher, research associate of the CRSN-Lwiro, Democratic Republic of Congo \\ ¥ Both authors contributed equally to the manuscript \\ *Corresponding author, email: francngera6@gmail.com
}

\begin{abstract}
The Albertine Rift is one of Africa's major biodiversity hotspots. However, the aquatic fauna of its western border in Democratic Republic of Congo (DRC) remains understudied and poorly characterised. Kahuzi-Biega National Park (KBNP) in DRC is a crucial link for biodiversity conservation of this hotspot; however, scientific studies of this rich biodiversity are scarce because of insecurity and harsh field conditions. In the current study, benthic macroinvertebrates communities and some environmental conditions were investigated in 10 rivers in the KBNP. Eighty-four morphospecies, belonging to 47 families and 12 orders, were sampled from 13 sites. The abundance and diversity of taxa within systematic groups varied across the study area. Spatial distribution of systematic groups was also analysed. The high morphospecies richness, characterised by the number of families and Shannon's and Simpson's Diversity Indices, recorded at some sites was probably owing to the diversity of substratum and undisturbed environmental conditions observed in these forested rivers. Conversely, some sites presented lower species richness, possibly because of unfavourable natural conditions.
\end{abstract}

Keywords: aquatic insects, aquatic invertebrates, biotic indices, conservation ecology, species diversity indices, water quality

Introduction

Across the world, many national agencies and nongovernmental organizations use aquatic macroinvertebrates as indicators of water quality because of their sensitivity to habitat modifications (Hussain and Pandit 2012) and pollutants (Chutter 1972; Rosenberg and Resh 1993; Dallas 1997; Abel 2002; Duran 2006; Ziglio et al. 2006), and the existence of well-established and cost-effective sampling procedures (Rosenberg and Resh 1993). The use of benthic macroinvertebrates as bioindicators has been developed in many studies of the relationships between benthic macroinvertebrate communities and environmental conditions, mainly in North America, Europe and more recently in South Africa, the Indian subregion and Asia (see Chutter 1972; Dallas 1997, 2005, 2007a, 2007b; Dicken and Graham 2002; Benstead and Pringle 2004; Dallas and Day 2004; Kasangaki et al. 2006; Kasangaki et al. 2008; Lorion and Kennedy 2009; Arthur 2010; Davies et al. 2010; and Hussain and Pandit 2012 for a synthetic review). The aquatic invertebrate fauna in central Africa, however, remains understudied. In this vast area, the Democratic Republic of Congo (DRC) occupies an important space.

For decades, insecurity and a critical lack of funding for Congolese research have prevented researchers from studying and analysing the aquatic invertebrate fauna in the streams and rivers of KBNP, which is located at the eastern border of the country and is recognised as a major biodiversity hotspot in the African Rift region. A few studies were carried out in rivers surrounding KBNP in the 1950s and 1960s, but these focused on physico-chemical characteristics (Marlier, 1954b) and on Trichoptera (Hydropsychidae) ecology and description (Marlier 1954a, 1961, 1962). Later, Statzner (1975, 1976), Jaquemart and Statzner (1981), and Kopelke (1979, 1981) studied the caddisflies and mayflies of some streams in the area. More recently, studies reported the presence of about 100 morphospecies of macroinvertebrates (Ngera et al. 2009) and two new species of Trichoptera (Pauls et al. 2010) in the rivers and streams of Lake Kivu basin, close to the KBNP border. Within the KBNP zone, some information was collected through the 'Programme Biodiversité des Écosystèmes Aquatiques et terrestres du Rift Albertin' (P-BEATRA 2005), which promoted collaboration between scientific institutions and conservation organizations to increase the biodiversity knowledge of the African Rift region. For instance, Bisimwa et al. (2013) provided a preliminary listing of epilithic algae and Bagalwa et al. (2012) characterised physico-chemical properties of watercourses in KBNP. Information on aquatic macroinvertebrate biodiversity in KBNP, however, remain sparse.

KBNP is currently flagged as 'in danger' by UNESCO on the list of World Heritage Sites (https://whc.unesco.org/fr/ soc/3812 [Accessed 1 February 2019]). The main threats 
to biodiversity include poaching, hunting, habitat conversion and fragmentation, agricultural practices (slash and burn), farms and illegal (and mainly artisanal) mining (https://whc. unesco.org/fr/soc/3812). It is therefore crucial to provide reliable scientific information about the biodiversity of this protected area to support conservation efforts for the natural capital of this region.

The current study provides initial information on the benthic invertebrate diversity of headwaters streams of KBNP and preliminary insights on the environmental conditions of these watercourses.

\section{Materials and methods}

\section{Study area}

Kahuzi-Biega National Park (KBNP; $1^{\circ} 36^{\prime} \mathrm{S}$ to $2^{\circ} 37^{\prime} \mathrm{S}$, $27^{\circ} 33^{\prime} \mathrm{E}$ to $28^{\circ} 46^{\prime} \mathrm{E}$ ) is a World Heritage Site (see UNESCO website at http://whc.unesco.org/en/list/137) located $20 \mathrm{~km}$ west of the city of Bukavu, South-Kivu province, Democratic Republic of Congo (Figure 1). The park is located on the western border of the Albertine Rift. Established in 1937 as a Zoological Forest Reserve of $600 \mathrm{~km}^{2}$, KBNP was given the status of National Park in 1970. Its initial area was extended to $6000 \mathrm{~km}^{2}$ by a presidential decree in July 1975 (Mutimanwa 2001; Kasereka 2003; UNEP-WCMC 2009). The shape of the park is complex, comprising two distinct areas that are joined by a $7.4-\mathrm{km}$ wide corridor (Figure 1). KBNP embraces a large variety of ecosystems and hosts a spectacular biodiversity with a high rate of endemism. Mühlenberg et al. (1995) estimated: 194 mammal species, including the eastern lowland gorilla; 349 bird species, 42 of which are endemic, and; at least 1178 described species of plants in and around the park.

The smaller $\left(600 \mathrm{~km}^{2}\right)$ eastern part of the park is characterised by high altitude mountains (ranging between 1800 and $3308 \mathrm{~m}$ asl) and is dominated by bamboo forest, primary and secondary mountain forests and swamp forests (Liengola 2008; Bagalwa et al. 2012). The larger (5 $400 \mathrm{~km}^{2}$ ) western part of the park embraces lower mountain rainforests (700 to $1700 \mathrm{~m}$ asl). The soils are of volcanic origin, and two extinct volcanoes, Kahuzi (3 $308 \mathrm{~m}$ asl) and Biega (2 $790 \mathrm{~m}$ asl), dominate the park's topography. In the highlands, mean temperatures vary between $10.4{ }^{\circ} \mathrm{C}$ and $18{ }^{\circ} \mathrm{C}$, whereas in the lowlands, they vary between $15{ }^{\circ} \mathrm{C}$ and $20^{\circ} \mathrm{C}$. The mean annual rainfall is $1900 \mathrm{~mm}$, with a short dry season from June to August and a long wet season from September to May (UNEP-WCMC 2009). The rivers and streams of the western area of the park flow into the Luka and Luhoho Rivers, which drain into the Lowa River, a main tributary of Lualaba River in the Congo Basin. The rivers and streams of the eastern area of the park drain into Lake Kivu, a reservoir on the Ruzizi River, which drains towards Lake Tanganyika. Figure 1 shows only the main rivers, because there is a paucity of geodata on rivers and streams of this region, and the dense vegetation cover of KBNP limits the efficient use of satellite imagery to map the rivers.

\section{Aquatic macroinvertebrate sampling}

Aquatic macroinvertebrates were sampled at 13 sites located on 10 headwater streams in the KBNP (Table 1).
Samples were collected between July and August in 2008 and 2010 (about two samples per week). Four sites were located on rivers draining eastwards into Lake Kivu: Chanderema $(\mathrm{CH})$, Langa (LNG) and Mushuva (MU_I, MU_II) Rivers. The remaining nine sites were located on rivers flowing westwards into the Congo Basin: Busangizi (BUS), Chumba (CHU), Cinya (CNY_I, CNY_II), Citori (Cl), Mirembo (MIR_I, MIR_II), Mugaba (MUG) and Nabugobugo (NBG) Rivers. The sites were selected based on accessibility and a variety of environmental conditions, such as canopy cover, the presence of aquatic and marginal vegetation and the nature of bottom substrate. One of the sites, CNY_II, exhibited characteristics 'typical' of a swamp site, namely slow current, decaying vegetation, trees growing in water and a low pH (Zoltai and Vitt 1995; Mackie 2004). Note that some of the common characteristics of a swamp zone, such as a strong chemical reducing environment (Barnaud and Fustec 2007), were not assessed in this study. The other 12 sites were all flowing water sites.

Macroinvertebrates were sampled using a $30-\mathrm{cm}$ wide D-frame net with a mesh of $500 \mu \mathrm{m}$. All available biotopes, such as stones, cobbles, gravel, debris, vegetation, sand, mud, aquatic and marginal vegetation, were sampled vigorously by kicking, turning and scraping using feet and hands. Dislodged macroinvertebrates were collected with the net. In addition, macroinvertebrates were hand-picked from stones and other substrates. As far as possible, in each site, similar sampling efforts were adopted. At each site, 10 minutes were allocated to collect aquatic organisms. To account for the relative coverage of different microhabitats, the total sampling time was divided between them. Accordingly, the time dedicated to sample each microhabitat was proportional to its percentage of coverage at that site. Environmental characteristics for the sampling stations are summarised in Table 2.

All collected organisms preserved in $70 \%$ alcohol on site, stored in plastic bottles and taken to the laboratory (CRSN/Lwiro, Lwiro, DRC) for identification. Specimens were sorted, counted and identified to the lowest taxonomic level possible, usually family or genus, and morphospecies where possible (morphospecies are taxa belonging to the same genera, but with different morphology and therefore likely to be different species), using dichotomous keys (Marlier 1962; De Moor et al. 2003a, 2003b; Tachet et al. 2003; Sunderman et al. 2007). Identification of some specimens was confirmed by specialists at Minnesota University, USA, and the Albany Museum, Department of Freshwater Invertebrates, Grahamstown, South Africa.

\section{Water chemistry sampling}

Temperature, acidity $(\mathrm{pH})$, conductivity and dissolved oxygen concentrations were recorded at a single point at each site in 2008 and 2010. Nutrient concentrations (nitrate as $\mathrm{NO}_{3}$ and phosphate as $\mathrm{PO}_{4}$ ) were measured only in 2008. Temperature $\left(T^{\circ}\right.$, expressed in degree Celsius), conductivity (expressed $\mu \mathrm{S} \mathrm{cm}^{-1}$, and related to the total dissolved salts concentration, or TDS) and water acidity (expressed on the $\mathrm{pH}$ scale) were measured with a HANNA Combo ${ }^{\circledR}$ multimeter (HI98129). Dissolved oxygen was measured using a HANNA dissolved oxygen 


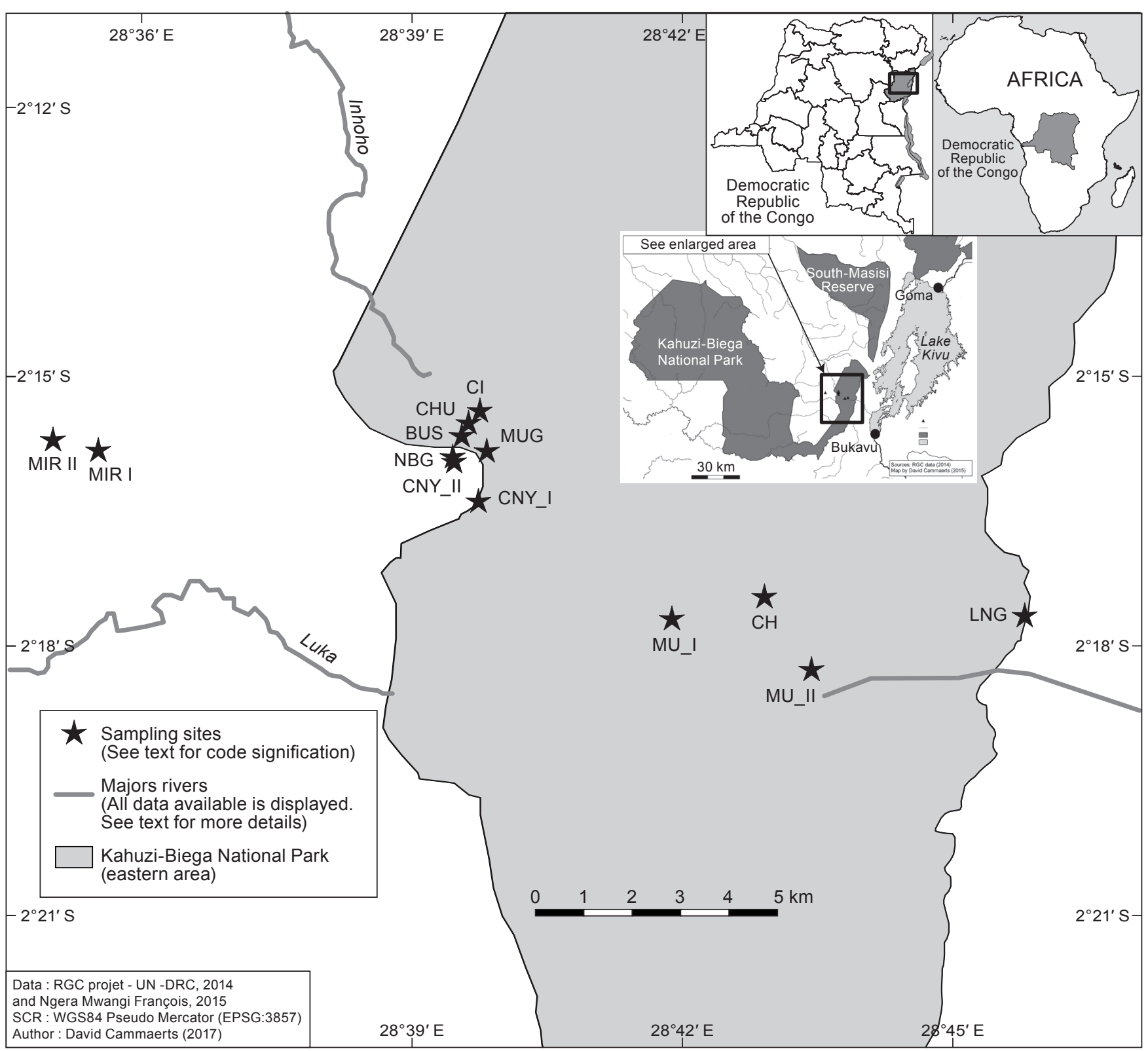

Figure 1: Sampling sites in the Kahuzi-Biega National Park (KBNP), eastern Democratic Republic of Congo

meter (HI91417). Water samples were collected in 2008 and nutrient concentrations were analysed in the CRSN/ Lwiro laboratory by colorimetric methods (see Bagalwa et al. 2012 for materials and methods). Water velocity was measured three times by timing a float as it moved over a 5-m or 10-m length of river (see Davies and Day 1998 for details) and the mean value calculated. Discharge was estimated from the product of current velocity and cross-sectional area of the river. Substrate composition of the river bed was expressed as a percentage of coverage of each category of substrate.

\section{Statistical analysis}

For each sampling site, relative abundance (\%) was calculated as the number of individuals belonging to a taxonomic group collected at a site divided by the total number of individuals collected for that site, multiplied by 100. For each sampling site, species richness (following Klemm et al. 1990), the Shannon-Wiener's Diversity Index (H') (Spellerberg and Fedor 2003) and the Simpson's Diversity Index (D) were calculated. Data for the two years (2008 and 2010) were pooled, and H' and D indices were calculated using family level data (Guerold 2000). A diversity $t$-test (Poole 1974) was used to compare species diversity between sites. No statistical analyses were done on the relative abundance, because of the non-standardised sampling procedure.

A principal component analysis (PCA), based on a variance-covariance matrix, was used to determine which of the measured environmental parameters (temperature, 
Table 1: Sampled sites in the Kahuzi-Biega National Park

\begin{tabular}{|c|c|c|c|c|c|c|}
\hline $\begin{array}{l}\text { Site name } \\
\text { (derived from river name) }\end{array}$ & Site code & River origin & Watershed & Latitude & Longitude & $\begin{array}{c}\text { Altitude } \\
\text { (metres above } \\
\text { sea level) }\end{array}$ \\
\hline Chanderema & $\mathrm{CH}$ & Civanga & Lac Kivu Basin & $2^{\circ} 17^{\prime} 25^{\prime \prime} \mathrm{S}$ & $28^{\circ} 43^{\prime} 00^{\prime \prime} \mathrm{E}$ & 2049 \\
\hline Mushuva I & MU_I & Bugulumiza & Lac Kivu Basin & $2^{\circ} 17^{\prime} 34^{\prime \prime} \mathrm{S}$ & $28^{\circ} 41^{\prime} 54^{\prime \prime} \mathrm{E}$ & 2175 \\
\hline Mushuva II & MU_II & Bugulumiza & Lac Kivu Basin & $2^{\circ} 18^{\prime} 12^{\prime \prime} \mathrm{S}$ & $28^{\circ} 43^{\prime} 37^{\prime \prime} \mathrm{E}$ & 2015 \\
\hline Langa & LNG & Civanga & Lac Kivu Basin & $2^{\circ} 17^{\prime} 35^{\prime \prime} \mathrm{S}$ & $28^{\circ} 45^{\prime} 24^{\prime \prime} \mathrm{E}$ & 1990 \\
\hline Nabugobugo & NBG & Musisi & Congo River Basin & $2^{\circ} 15^{\prime} 55^{\prime \prime} \mathrm{S}$ & $28^{\circ} 39^{\prime} 04^{\prime \prime} \mathrm{E}$ & 2161 \\
\hline Mirembo I & MIR_I & Mugaba & Congo River Basin & $2^{\circ} 16^{\prime} 01^{\prime \prime} \mathrm{S}$ & $28^{\circ} 35^{\prime} 44^{\prime \prime} \mathrm{E}$ & 2264 \\
\hline Mirembo II & MIR_II & Mugaba & Congo River Basin & $2^{\circ} 15^{\prime} 30^{\prime \prime} \mathrm{S}$ & $28^{\circ} 34^{\prime} 52^{\prime \prime} \mathrm{E}$ & NA \\
\hline Chumba & $\mathrm{CHU}$ & Mugaba & Congo River Basin & $2^{\circ} 15^{\prime} 16^{\prime \prime} \mathrm{S}$ & $28^{\circ} 39^{\prime 2} 22^{\prime \prime} \mathrm{E}$ & 2332 \\
\hline Citori & $\mathrm{Cl}$ & Mugaba & Congo River Basin & $2^{\circ} 15^{\prime} 11^{\prime \prime} \mathrm{S}$ & $28^{\circ} 39^{\prime} 44^{\prime \prime} \mathrm{E}$ & 2384 \\
\hline Cinya I & CNY_I & Mugaba & Congo River Basin & $2^{\circ} 16^{\prime} 40^{\prime \prime} \mathrm{S}$ & $28^{\circ} 39^{\prime} 52^{\prime \prime} \mathrm{E}$ & 2327 \\
\hline Cinya II & CNY_II & Mugaba & Congo River Basin & $2^{\circ} 16^{\prime} 08^{\prime \prime} S$ & $28^{\circ} 39^{\prime} 35^{\prime \prime} \mathrm{E}$ & 2303 \\
\hline Mugaba & MUḠ & Mugaba & Congo River Basin & $2^{\circ} 15^{\prime} 51^{\prime \prime} \mathrm{S}$ & $28^{\circ} 39^{\prime} 57^{\prime \prime} \mathrm{E}$ & 2289 \\
\hline Bwangizi & BWA & Mugaba & Congo River Basin & $2^{\circ} 15^{\prime} 33^{\prime \prime} \mathrm{S}$ & $28^{\circ} 39^{\prime} 12^{\prime \prime} \mathrm{E}$ & 2300 \\
\hline
\end{tabular}

$\mathrm{NA}=$ data not available

Table 2: Physical characteristics for the 13 sampling sites

\begin{tabular}{|c|c|c|c|c|c|c|c|c|c|c|c|c|}
\hline Site name & $\begin{array}{l}\text { Site } \\
\text { code }\end{array}$ & Stones & Cobbles & Pebbles & Gravel & Sand & Mud & Debris & $\begin{array}{c}\text { Aquatic and } \\
\text { Marginal } \\
\text { vegetation }\end{array}$ & $\begin{array}{c}\text { Canopy } \\
\text { cover }\end{array}$ & $\begin{array}{c}\text { Flow } \\
\left(\mathrm{m}^{3} \mathrm{~s}^{-1}\right)\end{array}$ & $\begin{array}{l}\text { velocity } \\
\left(\mathrm{cm} \mathrm{s}^{-1}\right)\end{array}$ \\
\hline Chanderema & $\mathrm{CH}$ & $10 \%$ & - & $5 \%$ & $60 \%$ & $15 \%$ & - & $10 \%$ & - & $100 \%$ & 0.015 & 8.03 \\
\hline Mushuva I & MU_I & - & $15 \%$ & $60 \%$ & $5 \%$ & $5 \%$ & $5 \%$ & $10 \%$ & - & $100 \%$ & 0.100 & 20.05 \\
\hline Mushuva II & MU_II & - & $5 \%$ & - & $75 \%$ & - & $5 \%$ & $10 \%$ & $5 \%$ & $100 \%$ & 0.100 & 52.00 \\
\hline Langa & LNG & $10 \%$ & $35 \%$ & $40 \%$ & $10 \%$ & - & - & $5 \%$ & - & $100 \%$ & 0.088 & 42.02 \\
\hline Nabugobugo & NBG & $65 \%$ & $5 \%$ & $10 \%$ & - & $15 \%$ & - & $5 \%$ & - & $100 \%$ & 0.074 & 36.04 \\
\hline Mirembo I & MIR_I & - & - & - & $85 \%$ & $5 \%$ & $5 \%$ & $5 \%$ & - & $100 \%$ & 0.030 & 22.13 \\
\hline Mirembo II & MIR_II & $15 \%$ & $5 \%$ & $5 \%$ & $25 \%$ & $5 \%$ & $5 \%$ & $40 \%$ & - & $100 \%$ & 0.030 & 22.00 \\
\hline Chumba & $\mathrm{CHU}$ & - & - & $5 \%$ & $45 \%$ & - & - & $10 \%$ & $40 \%$ & $100 \%$ & 0.058 & 36.00 \\
\hline Citori & $\mathrm{Cl}$ & - & - & - & - & $40 \%$ & $40 \%$ & $20 \%$ & - & $100 \%$ & 0.021 & 31.02 \\
\hline Cinya I & CNY_I & - & $10 \%$ & $40 \%$ & $30 \%$ & - & $5 \%$ & $15 \%$ & - & $100 \%$ & 0.036 & 25.02 \\
\hline Cinya II & CNY_II & - & - & - & - & - & $20 \%$ & $75 \%$ & $5 \%$ & $100 \%$ & NA & NA \\
\hline Mugaba & $M U \bar{G}$ & - & - & - & $80 \%$ & $5 \%$ & $5 \%$ & $5 \%$ & $5 \%$ & $100 \%$ & 0.110 & 36.02 \\
\hline Bwangizi & BWA & - & - & - & $90 \%$ & $5 \%$ & - & $5 \%$ & - & $100 \%$ & 0.740 & 51.01 \\
\hline
\end{tabular}

$\mathrm{NA}=$ data not available

$\mathrm{pH}$, conductivity, dissolved oxygen, nitrate, phosphate discharge, as well as water velocity) had the strongest relationship with benthic macroinvertebrate communities collected at each site. All data were $\log _{10}(x+1)$ transformed prior to analysis to achieve normality of the data. The following water parameters were included in this analysis: 2008 and 2010 data for temperature, $\mathrm{PH}$, conductivity and dissolved oxygen and 2008 data for nutrients. An iterative calculation was used to take into account the non-available data. The canopy cover parameter was excluded from this analysis. For each axis the eigenvalues and the percentage of total variance were evaluated.

Cluster analyses, based on Euclidean distance and Simpson's Diversity Index were used in addition to PCA to evaluate the relationship between environmental parameters and benthic macroinvertebrate communities at the sites. Basic graphical analysis of relative abundance were carried out to provide some insight on the trends in numerical dominance of main taxonomic groups at each site. All calculations and statistical analysis were done using the PAST V2.17 software (https://folk.uio.no/ohammer/past/).
Table 3: Chemical parameters recorded in the 13 sampling sites. For $\mathrm{NO}_{3}$ and $\mathrm{PO}_{4}$, measurement were only made in 2008. All other parameters were measured in 2008 and 2010

\begin{tabular}{|c|c|c|c|c|c|c|}
\hline Sites & $\begin{array}{c}\mathrm{T} \\
\left({ }^{\circ} \mathrm{C}\right)\end{array}$ & $\mathrm{pH}$ & $\begin{array}{l}\text { Cond. } \\
(\mu S)\end{array}$ & $\begin{array}{c}\mathrm{DO} \\
\left(\mathrm{mg} \mathrm{l}^{-1}\right)\end{array}$ & $\begin{array}{c}\mathrm{NO}_{3} \\
\left(\mathrm{mg} \mathrm{l}^{-1}\right)\end{array}$ & $\begin{array}{c}\mathrm{PO}_{4} \\
\left(\mathrm{mg} \mathrm{l}^{-1}\right)\end{array}$ \\
\hline$\overline{\mathrm{CH}}$ & 14.1 & 6.1 & 54 & 7.60 & 0.0217 & 0.0523 \\
\hline MU_I & 11.3 & 7.4 & 64 & 6.20 & 0.0198 & 0.0276 \\
\hline MU_II & 13.0 & 7.6 & 69 & 6.11 & NA & NA \\
\hline BUS & 13.8 & 6.1 & 13 & 5.68 & 0.0192 & 0.0082 \\
\hline MI_I & 13.2 & 6.5 & 19 & 5.80 & 0.0192 & 0.0086 \\
\hline MI_II & 13.3 & 6.3 & 17 & 6.10 & NA & NA \\
\hline LNG & 14.9 & 7.0 & 17 & 7.55 & 0.0136 & 0.0105 \\
\hline $\mathrm{CHU}$ & 14.6 & 5.6 & 13 & 5.70 & 0.0173 & 0.0114 \\
\hline $\mathrm{Cl}$ & 14.5 & 5.5 & 14 & 5.38 & 0.0155 & 0.0105 \\
\hline CNY_I & 13.2 & 7.6 & 8 & 3.50 & NA & NA \\
\hline CNY_II & 13.5 & 5.7 & 15 & 1.89 & 0.0217 & 0.0238 \\
\hline MUG & 13.7 & 6.9 & 30 & 3.37 & 0.0173 & 0.0162 \\
\hline NBG & 13.9 & 5.0 & 13 & 5.79 & 0.0155 & 0.0124 \\
\hline
\end{tabular}

$\mathrm{NA}=$ data not available 


\section{Results}

\section{Physical and chemical parameters}

Water temperature varied between $11.3{ }^{\circ} \mathrm{C}$ (MU_I) and $14.9{ }^{\circ} \mathrm{C}$ (LNG) (Table 3). $\mathrm{pH}$ values varied between 5.0 (NBG) and 7.6 (CNY_I). Conductivity recorded during the sampling periods ranged between $8.0 \mu \mathrm{S} \mathrm{cm}^{-1}$ (CNY_I) and $69.0 \mu \mathrm{S} \mathrm{cm} \mathrm{cm}^{-1}$ (MU_II). Dissolved oxygen varied between $1.9 \mathrm{mg} \mathrm{I}^{-1}\left(\mathrm{CNY}\right.$ II) and $7.6 \mathrm{mg} \mathrm{I}^{-1}(\mathrm{CH})$. In comparison with Lake Kivu tributaries, most of the Congo River tributaries showed lower dissolved oxygen levels, ranging between $1.9 \mathrm{mg} \mathrm{l}^{-1}$ and $6.1 \mathrm{mg} \mathrm{l}^{-1}$. Nitrate concentration was the highest at $\mathrm{CH}\left(0.022 \mathrm{mg} \mathrm{l}^{-1}\right)$, whereas the lowest concentration $\left(0.014 \mathrm{mg} \mathrm{l}^{-1}\right)$ was

Table 4: PCA eigenvalues of environmental parameters based on a variance-covariance matrix

\begin{tabular}{llc}
\hline Principal component & Eigenvalue & Percentage variance \\
\hline 1 & 0.0924 & 61.258 \\
2 & 0.0362 & 24.014 \\
3 & 0.0151 & 9.989 \\
4 & 0.0039 & 2.589 \\
5 & 0.0014 & 0.903 \\
6 & 0.0012 & 0.811 \\
7 & 0.0004 & 0.296 \\
8 & 0.0002 & 0.139 \\
\hline
\end{tabular}

recorded at LNG. The highest phosphate concentration was recorded at $\mathrm{CH}\left(0.052 \mathrm{mg} \mathrm{l}^{-1}\right)$, whereas the lowest concentration was recorded at BUS $\left(0.008 \mathrm{mg} \mathrm{l}^{-1}\right)$.

In the PCA of environmental parameters, the Jolliffe cut-off test set the important components higher than 0.028 , and the first two axes combined (PC1 $=0.25$ and PC2 $=0.08$ ) explained more than $95 \%$ of the total variability in the data. Table 4 shows the importance of each of the eight principal components. The PCA (Figure 2) showed a similarity between $\mathrm{CH}$ and $\mathrm{MU}$ I, which both flow into Lake Kivu. A second group was formed by streams primarily draining into the Congo Basin. The MU_II site was separated from the eastward flowing sites in the PCA scatter diagram, probably owing to the high water velocities at this site relative to the other sites. The strongest explanatory variable of the spatial distribution of all sampling sites was current velocity, with conductivity also appearing as a strong explanatory variable.

In the cluster analysis (based on Euclidean distance; Figure 3), Cluster B (CNYI_II site) strongly diverged from the other sites, with the sites, with the exception of CNY_II, grouping in Cluster A. In subgroup A1, a similarity was found between $\mathrm{CH}$ and $\mathrm{MU}$ _. Both sites were also similar to MU_II as the Chanderema River $(\mathrm{CH})$ drains into the Mushuva River, which in turn flows into Lake Kivu. The second subgroup (A2) was primarily formed by streams draining into the Congo Basin. The

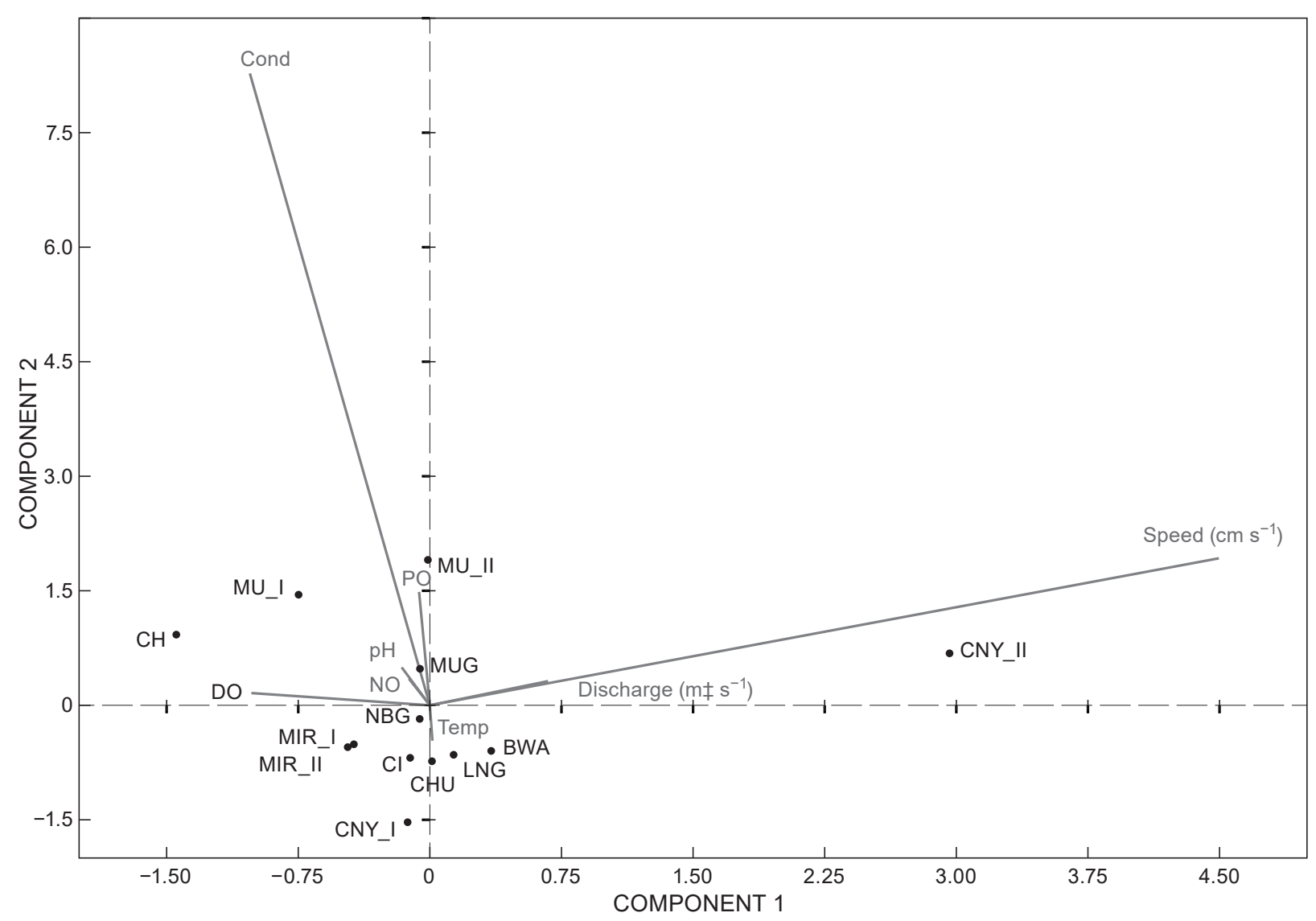

Figure 2: Scatter diagram of the PCA of environmental parameters based on a variance-covariance matrix. 


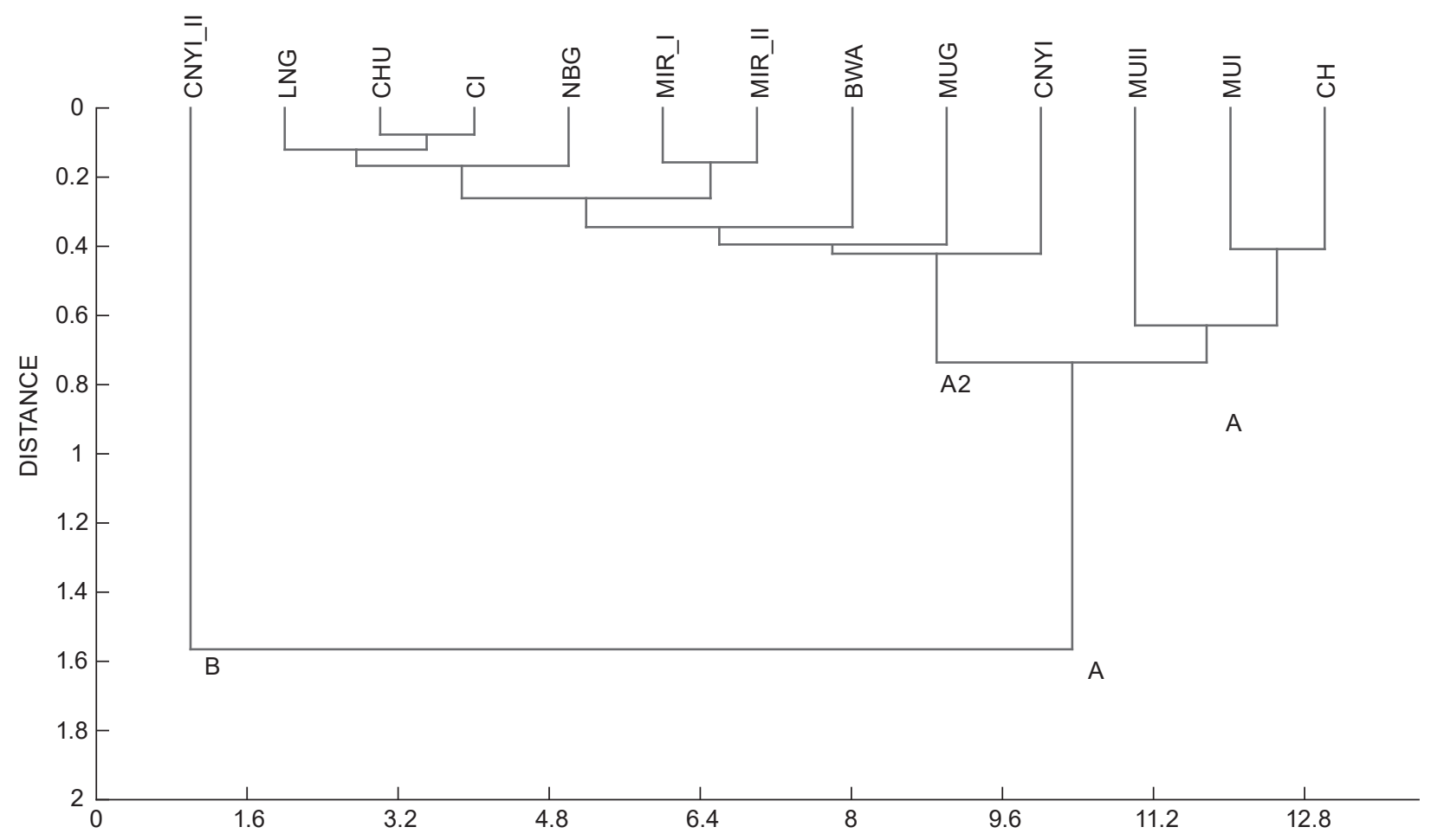

Figure 3: Cluster analysis of environmental parameters based on Euclidian distance

Table 5: List of aquatic macroinvertebrates sampled from Kahuzi-Biega National Park Rivers and streams. Abundance of specimens are rated as: $(+)=1$ to $9 ;(++)=10$ to $99 ;(+++)=\geq 100$ specimens.

\begin{tabular}{|c|c|c|c|c|c|c|c|c|c|c|c|c|c|c|c|c|}
\hline Order & Family & Genus & Morphospecies & $\mathrm{CH}$ & MU_I & MU_II & BUS & MIR_I & MIR_II & LNG & $\mathrm{CHU}$ & $\mathrm{Cl}$ & CNY_I & CNY_II & MUG & NBG \\
\hline \multirow[t]{12}{*}{ Ephemeroptera } & Leptophlebiidae & Adenophlebia & Adenophlebia sp1 & ++ & +++ & +++ & ++ & +++ & +++ & ++ & ++ & ++ & + & & ++ & ++ \\
\hline & & & Adenophlebia sp2 & ++ & ++ & ++ & ++ & ++ & +++ & ++ & ++ & + & & & + & ++ \\
\hline & & & Adenophlebia sp3 & + & & & & & & & & & & & & \\
\hline & Baetidae & Baetis & Baetis sp1 & ++ & +++ & +++ & + & ++ & + & ++ & + & & & & & + \\
\hline & & & B. $\mathrm{sp} 2$ & + & & & & & & & & & & & & \\
\hline & & Centroptilum & Centroptilum sp. & & ++ & + & & & & & & & & & & \\
\hline & & Acentrella & Acentrella sp. & & & & & & & + & & & & & & \\
\hline & Heptageniidae & Afronurus & Afronurus sp. & + & + & + & + & & + & ++ & & & & & & \\
\hline & Dicercomyzidae & Dicercomyzon & Dicercomyzon sp. & + & & & & & + & & & & & & & + \\
\hline & Caenidae & Caenis & Caenis sp1 & & ++ & ++ & & + & + & & & & & & & \\
\hline & & & Caenis sp2 & & ++ & + & & & & & & & & & & \\
\hline & Prosopistomatidae & Binoculus (?) & Binoculus sp (?) & + & + & ++ & + & & ++ & & & & & & & \\
\hline \multirow[t]{11}{*}{ Diptera } & Unidentified & & Diptera Fam. gen. sp. & + & & & & & & & & & & & & \\
\hline & Tipuliidae & Tipula & Tipula sp. & & & & ++ & + & + & + & + & ++ & + & & + & + \\
\hline & & & Hexatoma sp. & + & & + & + & ++ & ++ & + & & + & & & + & \\
\hline & Ceratopogonidae & Ceratopogonidae gen. & Ceratopogonidae. gen. sp. & & & & + & & & & & & & +++ & & \\
\hline & Chironomiidae & Chironomiidae gen. & Chironomidae gen. sp. & & +++ & + & & + & ++ & + & ++ & & ++ & +++ & & \\
\hline & Limoniidae & Limoniidae gen. & Limoniidae gen. sp. & & & + & & & & & + & & & & & \\
\hline & Athericidae & Atrhichops & Atrhichops sp. & + & & & & + & & + & + & & & & & \\
\hline & & Atherix & Atherix sp. & & + & ++ & + & & & + & + & + & + & & + & ++ \\
\hline & Anthomiidae & Anthomiidae gen. & Anthomiidae gen. sp. & + & & & & & & & & & & & & \\
\hline & Simuliidae & Simulium & Simulium sp. & ++ & ++ & ++ & + & + & & + & & + & ++ & & & + \\
\hline & Dixidae & Dixa & Dixa sp. & & + & & & & & & & & & & & \\
\hline \multirow[t]{8}{*}{ Coleoptera } & Dytiscidae & Dytiscidae gen. & Dytiscidae gen. sp. & & + & & & + & & & & & & & & \\
\hline & Elmidae & Elmis & Elmis sp. & & & + & & & & & & & & & + & + \\
\hline & & Dupophilus & Dupophilus sp. & + & & + & & & & + & & & & & & \\
\hline & & Limnius & Limnius sp. & & & + & & & & & & + & & & & \\
\hline & & Riolus & Riolus sp. & & + & & & & & & & & & & & \\
\hline & Scirtidae & Hydrocyphon & Hydrocyphon sp. & & + & ++ & & + & + & ++ & & & ++ & & & ++ \\
\hline & & Elodes & Elodes sp. & & & + & + & & + & + & & & + & & & \\
\hline & Psephenidae & Eubrianax & Eubrianax sp. & & & + & & & + & ++ & + & & & & & \\
\hline
\end{tabular}


Table 5: (cont.)

\begin{tabular}{|c|c|c|c|c|c|c|c|c|c|c|c|c|c|c|c|c|}
\hline Order & Family & Genus & Morphospecies & $\mathrm{CH}$ & MU_I & MU_II & BUS & MIR_I & MIR_II & LNG & $\mathrm{CHU}$ & $\mathrm{Cl}$ & CNY_I & CNY_II & MUG & NBG \\
\hline & & Psephenidae gen. & Psephenidae gen. sp. & & & & & + & & & & & & & & \\
\hline & Gyrinidae & Dineutus & Dineutus sp. & & & & + & + & + & & & & & & & \\
\hline & & Aulonogyrus & Aulonogyrus sp. & & & & & + & + & & & & + & & & + \\
\hline & & Gyrinidae gen. & Gyrinidae gen. sp. & & & & & + & + & & + & + & & & & \\
\hline & & Gyrinus & Gyrinus sp. & & & & & & + & & & & & & & \\
\hline & Dryopidae & Dryopidae gen. & Dryopidae gen. sp. & & & & & & & & & & + & & & \\
\hline Plecoptera & Perliidae & Neoperla & Neoperla sp. & + & & + & ++ & ++ & ++ & ++ & + & ++ & & & ++ & ++ \\
\hline \multirow[t]{3}{*}{ Heteroptera } & Veliidae & Rhagovelia & Rhagovelia sp. & & & & & & & & + & ++ & & & & \\
\hline & Pleidae & Plea & Plea sp. & & & & & & & + & & & & & & \\
\hline & Nocteridae & Nocteridae gen. & Nocteridae gen. sp. & + & & & & & & & & & & & & \\
\hline \multirow[t]{21}{*}{ Trichoptera } & Hydropsychidae & Diplectronella & Diplectronella sp. & + & & & & & & & & & & & & \\
\hline & & $\begin{array}{l}\text { Ethiopsyche } \\
\text { (syn. Cheumatopsyche) }\end{array}$ & Ethiopsyche (Ch. sp.) & ++ & +++ & ++ & ++ & + & + & ++ & & ++ & ++ & & + & + \\
\hline & & Hydropsyche & Hydropsyche sp. & & + & & & + & + & + & & + & & & & \\
\hline & & Cheumatopsyche & Cheumatopsyche & & & & t++ & ++ & ++ & ++ & ++ & ++ & & & & \\
\hline & & Leptonema & Leptonema sp. & & & & & & & & & & & & ++ & ++ \\
\hline & Lepidostomatidae & Lepidostoma & Lepidostoma sp. & + & ++ & ++ & + & + & + & ++ & + & +++ & + & & + & t+ \\
\hline & Dipseudopsidae & Dipseudopsis sp. & Dipseudopsis sp. & ++ & & & + & + & & & & & + & & & \\
\hline & & Pseudoneureclipsis sp. & Pseudoneureclipsis sp. & + & & & & & + & + & & & & & & \\
\hline & Leptoceridae & Triaenodes & Triaenodes sp. & + & & & & & & & & & & & & \\
\hline & & Athripsodes & Athripsodes sp. & & & & & + & + & & & & & & & \\
\hline & & Oecetis & Oecetis sp. & + & & & & + & & & & & & & & \\
\hline & Glossosomatidae & Cryptothrix & Cryptothrix sp1 & & + & + & & & & & & & & & & \\
\hline & & & Cryptothrix sp2 & & + & & & & & & & & & & & \\
\hline & Polycentropodidae & Nyctiophylax & Nyctiophylax & & + & + & + & + & & & + & & & & & \\
\hline & & Cyrnus & Cyrnus sp. & & & & & & & & + & + & & & & \\
\hline & & Polycentropus & Polycentropus sp. & & & & & & & & & & + & & & \\
\hline & Philopotamidae & Chimarra & Chimarra sp. & & & + & & & & + & & + & + & & + & \\
\hline & & Thylakion & Thylakion sp. & & & & + & + & + & & + & & + & & + & \\
\hline & Pisuliidae & Pisula & Pisula sp. & & & & & & & & & & + & & & \\
\hline & Psychomyiidae & Tinodes & Tinodes sp. & & & & & + & & & & & & & & \\
\hline & Calamoceratidae & Anisocentropus & Anisocentropus sp. & & & & ++ & + & ++ & & ++ & + & & & + & + \\
\hline \multirow[t]{14}{*}{ Odonata } & Chlorocyphidae & Chlorocypha & Chlorocypha sp. & & & ++ & & & & & + & + & + & & + & \\
\hline & Aeshnidae & Aeshnidae gen. & Aeshnidae gen. & & & + & + & + & + & & & + & + & & + & + \\
\hline & & Anaciaeschna & Anaciaeschna & & & & & & & & & + & & & & + \\
\hline & & Boyera & Boyera & & & & + & + & + & & + & & + & & & \\
\hline & & Brachytron & Brachytron & & & & & & & & + & & & & & \\
\hline & Libellulidae & Brachytnemis & Brachytnemis & & & + & & & & & & & & & & \\
\hline & & Libellula & Libellula & & & & & & + & & & & & & & \\
\hline & & Sympetrum & Sympetrum & & & & & & & & & + & + & & & \\
\hline & Cordulidae & Cordulia & Cordulia & & & & ++ & & & + & & & & & + & \\
\hline & & Somatochlora & Somatochlora & & & & & + & + & & + & + & & & & ++ \\
\hline & Gomphidae & Gomphus & Gomphus sp. & & & & & + & + & & ++ & & & & + & + \\
\hline & & Paragomphus & Paragomphus sp. & & & & & + & & + & + & & & & ++ & + \\
\hline & & Onychogomphus & Onychogomphus sp. & & & & & & & & & & & & + & \\
\hline & Coenagrionidae & Coenagrionidae gen & Coenagrionidae gen. sp. & & & & & & + & & & & & & & \\
\hline Nematoda & Gordiacae & Gordiacae gen. & Gordiacae gen. sp. & & & & & & + & + & & + & & & & \\
\hline Bivalvia & Dreissenidae & Pisidium & Pisidium sp. & & & & & & & + & & & & & & \\
\hline Crustacea & Potamonidae & Potamonidae gen. & Potamonidae gen. sp. & ++ & ++ & ++ & + & + & + & ++ & & & & & ++ & + \\
\hline Turbellaria & Dugesiidae & Dugesia & Dugesia sp. & ++ & ++ & & & & & & & & & & & \\
\hline \multirow[t]{10}{*}{ Hirudinea } & Glossiphonidae & Theronmyson & Theronmyson sp. & & + & & & & & & & & & & & \\
\hline & & Helobdella & Helobdella sp. & & + & & + & & + & & & & + & ++ & & + \\
\hline & & Haementeria & Haementeria sp. & & & & & & & & & & + & + & & \\
\hline & Erpobdeliidae & Erpobdeliidae gen. & Erpobdeliidae gen. sp. & & & & & & & & & & + & + & & \\
\hline & & & Nbr morphospecies & 25 & 25 & 29 & 25 & 33 & 35 & 28 & 24 & 23 & 23 & 5 & 20 & 22 \\
\hline & & & Nbr genus & 21 & 22 & 27 & 24 & 32 & 34 & 27 & 23 & 22 & 22 & 4 & 19 & 21 \\
\hline & & & Nbr Families & 18 & 19 & 24 & 21 & 25 & 27 & 22 & 20 & 19 & 20 & 4 & 15 & 18 \\
\hline & & & Abundance (\%) & 7.08 & 27.39 & 9.85 & 8.09 & 5.23 & 11.4 & 6.05 & 3.88 & 5.91 & 3.82 & 2.71 & 3.7 & 4.82 \\
\hline & & & Shannon_H & 2.944 & 2.944 & 3.135 & 3.045 & 3.219 & 3.296 & 3.091 & 3 & 2.89 & 2.996 & 1.39 & 2.639 & 2.89 \\
\hline & & & Simpson_1-D & 0.9474 & 0.9474 & 0.957 & 0.952 & 0.96 & 0.963 & 0.955 & 0.95 & 0.94 & 0.95 & 0.75 & 0.929 & 0.944 \\
\hline
\end{tabular}


highest level of similarity was found between $\mathrm{CHU}$ and $\mathrm{Cl}$ in subgroup $\mathrm{A} 2$.

\section{Aquatic macroinvertebrates}

\section{Relative abundance and diversity}

Five thousand and forty specimens were collected at the 13 sites (Table 5). These belonged to 84 morphospecies (for a total of 77 identified genera), 47 families and 12 orders. The relative abundance varied between taxonomic groups (Figure 4). The most abundant orders were Ephemeroptera (40.2\% of specimens) and Trichoptera (25.8\% of specimens). Nematodes $(0.1 \%)$ and bivalves $(0.01 \%)$ were the least abundant. For most taxonomic groups, the numbers were made up from one or two dominant families. For instance, Ephemeroptera were dominated by Leptophlebiidae $(61.46 \%$ of collected specimens of this family) and Baetidae (28.97\%); Trichoptera by Hydropsychidae (63.66\%); Diptera by Chironomidae (mostly Tendipendini Chironomid Tribe;
$54.69 \%$ ), and; Odonata by Aeshnidae (27.53\%) and Gomphidae (26.57\%).

In terms of number of morphospecies recorded within each order (Figure 5), Trichoptera had the most morphospecies (21) followed by Odonata and Coleoptera (14 each), Diptera (13) and Ephemeroptera (12). The fewest morphospecies were found in the Order Plecoptera, the Order Decapoda, the Class Turbellaria, the Class Bivalvia (Order Veneroidea) and the Phylum Nematoda.

\section{Spatial distribution}

Spatial distribution of taxonomic groups showed two trends (Figure 6). Some groups (e.g. Diptera, Ephemeroptera and Trichoptera) were recorded at all sites, except CNY II. By contrast, Bivalvia, Nematoda, and Turbellaria occurred at one or two sites only.

The spatial distribution of morphospecies exhibited similar trends than those observed for the superior taxonomic levels. Many morphospecies, such as Adenophlebia sp. (Ephemeroptera) and Cheumatopsyche (Ethiopsyche) sp.

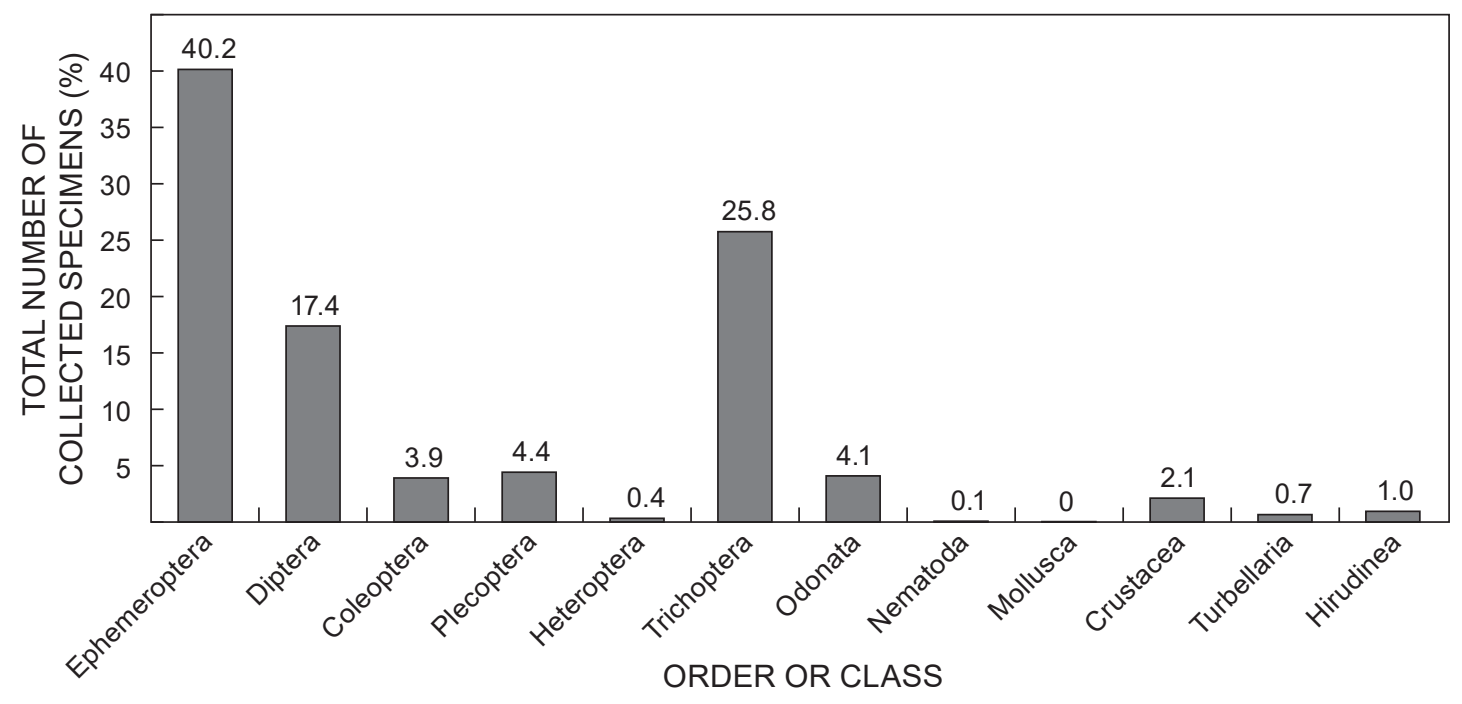

Figure 4: Relative abundance of collected order, expressed as a percentage of the total number of collected specimens/individuals

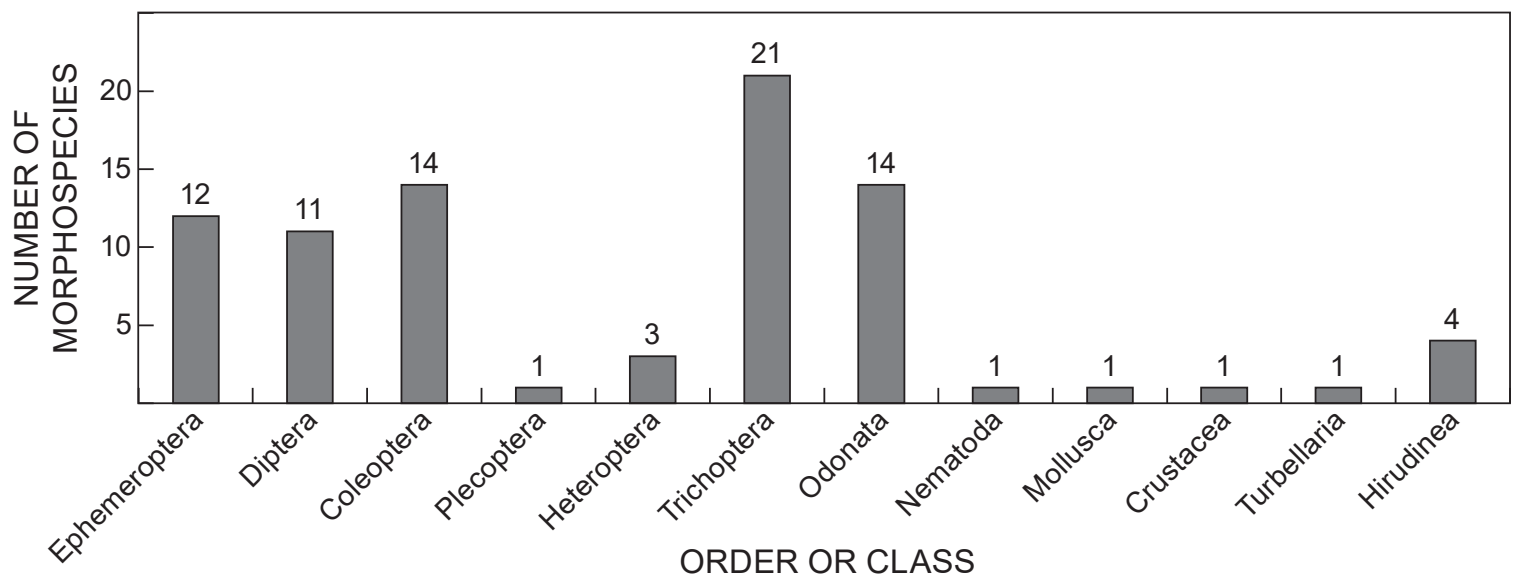

Figure 5: Number of morphospecies within each order or class 
(Trichoptera) were recorded at all sites, except those in the Cinya River (CNY_I and II) and in the Chumba Rivers (CHU), respectively. Other morphospecies, such as Caenis sp2 (MU_I and II), Leptonema sp. (MUG, NBG), Diplectronella sp. (CH), Acentrella sp. (LNG) and Plea sp. (LNG), only occurred at a few sites.

\section{Morphospecies richness}

Morphospecies richness also varied among the sites (Figure 7). The greatest number of taxa (35 morphospecies) was recorded at MIR_II and the least (5 morphospecies) at CNY_II.

The cluster analysis of site similarity (based on Simpson's Diversity Index calculated with morphospecies data), revealed the greatest similarity between sites in the same river or catchment (Figure 8). Cluster A, which comprised CNY_I and CNY_II, both on the Cinya River (CNY II with the characteristics of a swamp site), had the lowest diversity value (1.39). Cluster B was divided into two subgroups (B1 and $\mathrm{B} 2$ ) where $\mathrm{CH}$ separated from other sites. Subgroup B1 comprised MU_I and MU_II in the Mushuva River, and subgroup B2 grouped MIR_- and MIR II, in the Mirembo River, and Busangizi River site.

The number of families recorded varied among sites (Figure 9) (data pooled for 2008 and 2010 samples). The highest number of families was recorded at MIR_II (27 families). The lowest number was recorded

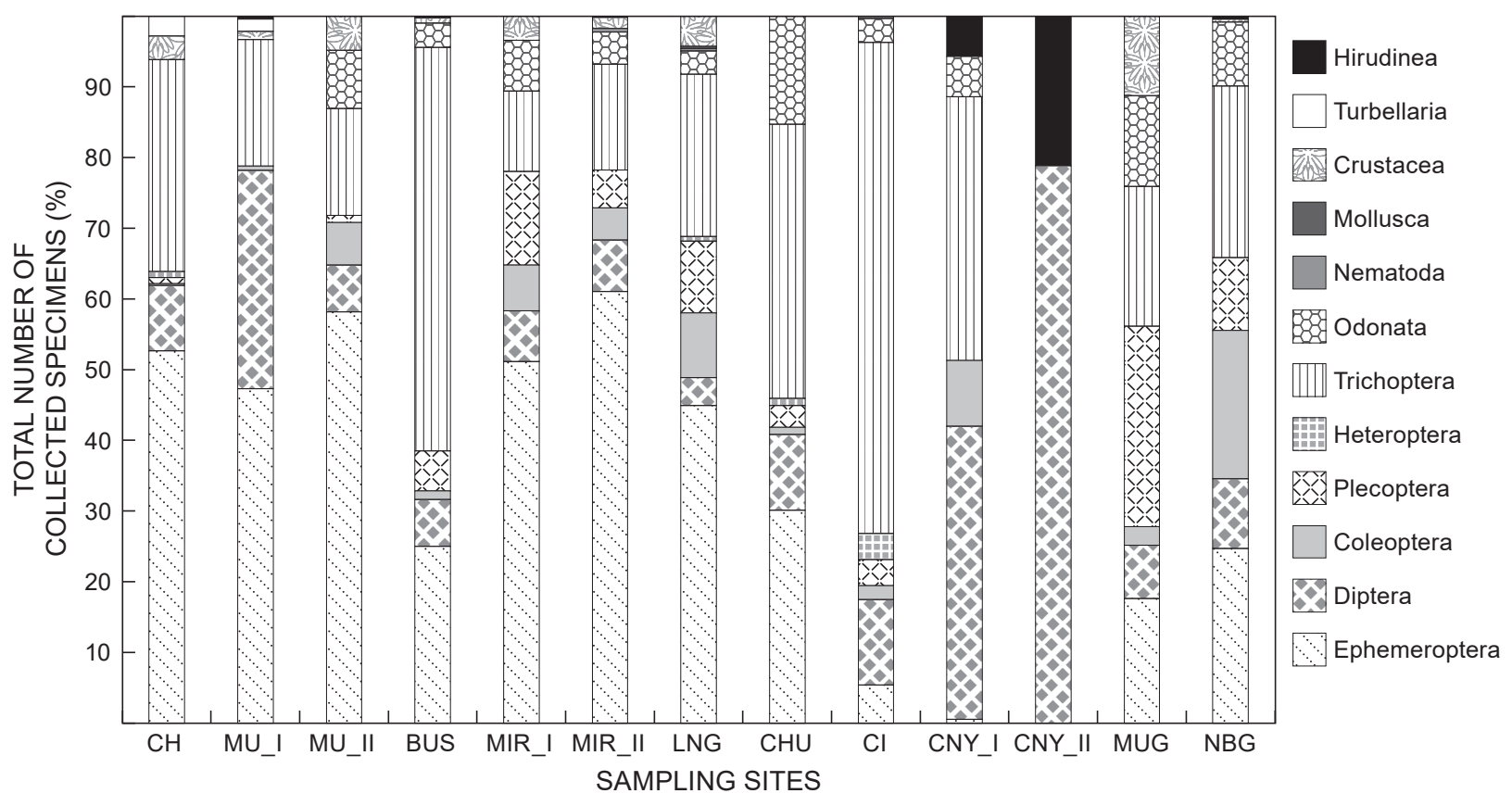

Figure 6: Spatial distribution of inventoried main taxonomic groups

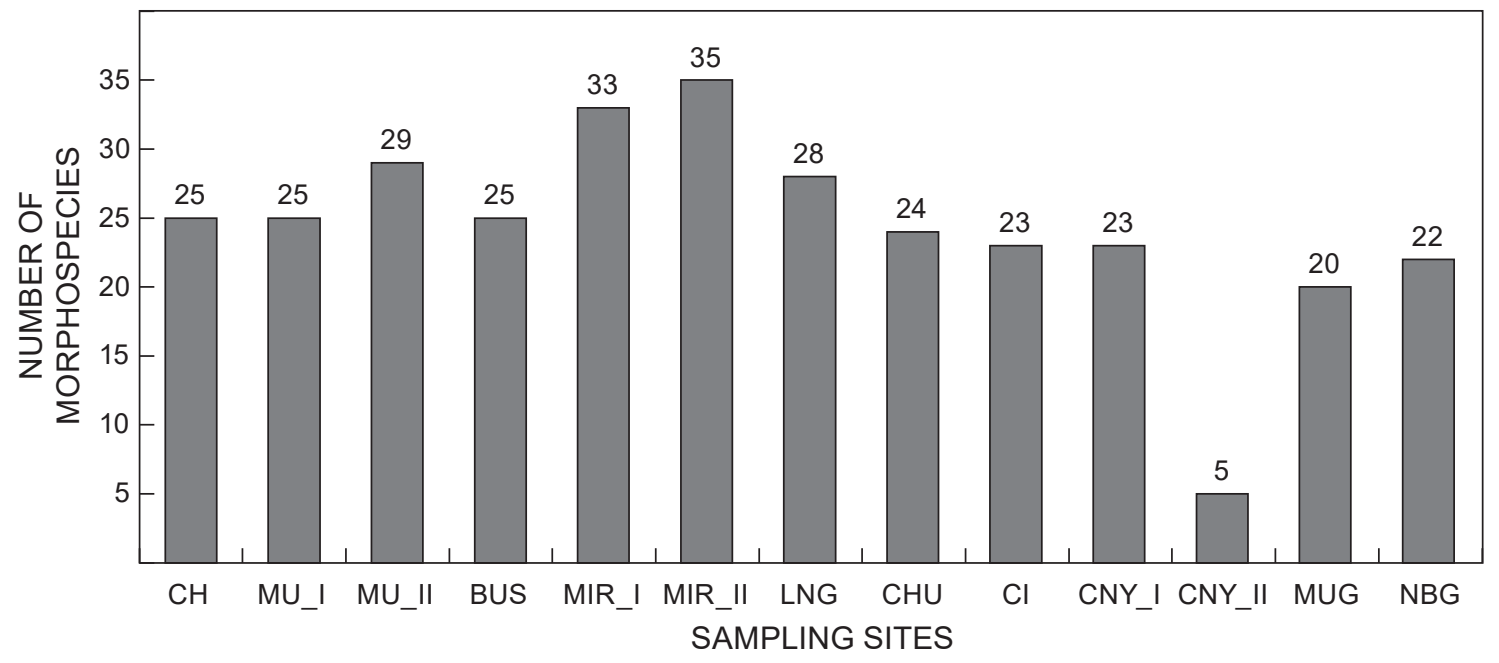

Figure 7: Morphospecies richness at sampled sites 
at CNY_II (4 families). which was significantly different (ANOVA, $p<0.0001$ ) from the other sites.

\section{Shannon-Wiener's Diversity Index}

MIR_II had the highest Shannon-Wiener's Diversity Index value (calculated at the family taxonomic level) (3.30) and was significantly different $(p<0.05)$ from CNY_II, which had the lowest value (1.39) (Figure 10). A $t$-test performed on the Shannon-Wiener's Diversity Indices also showed significant differences between CNY_II and the other sampling sites.

\section{Discussion}

\section{Abiotic conditions}

The low conductivity at sampling sites could be the result of multiple drivers, whose identification goes beyond the scope of the current study. Previous research supports the hypothesis that riparian vegetation improves ecological functions and quality of water of running watercourses. The presence of vegetation (especially forest cover like trees and shrubs) along and above the edge of a river can lead to low conductivity, low turbidity, low temperature

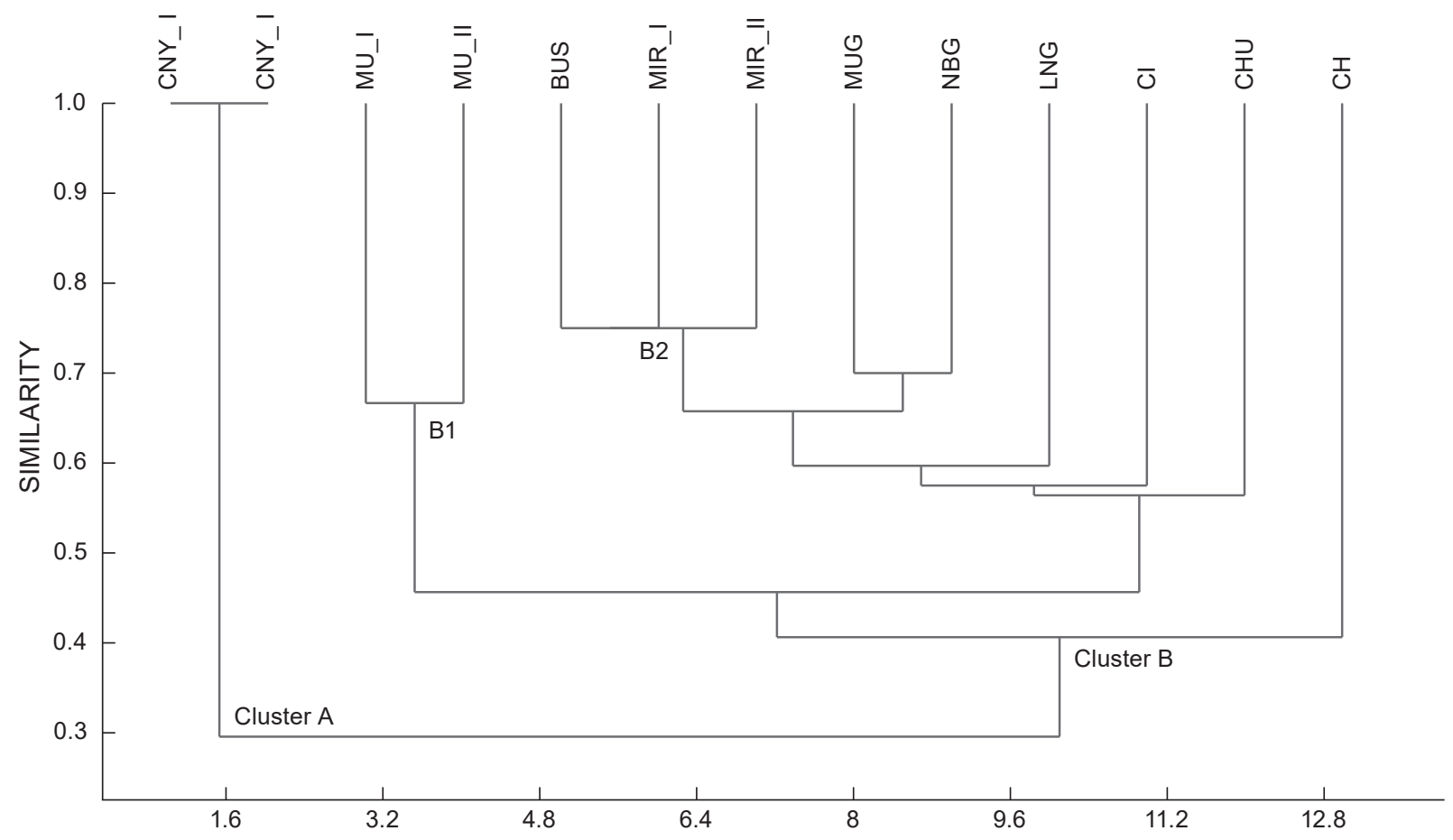

Figure 8: Cluster analysis based on Simpson's Diversity Index for 13 sites located in 10 streams, Kahuzi-Biega National Park

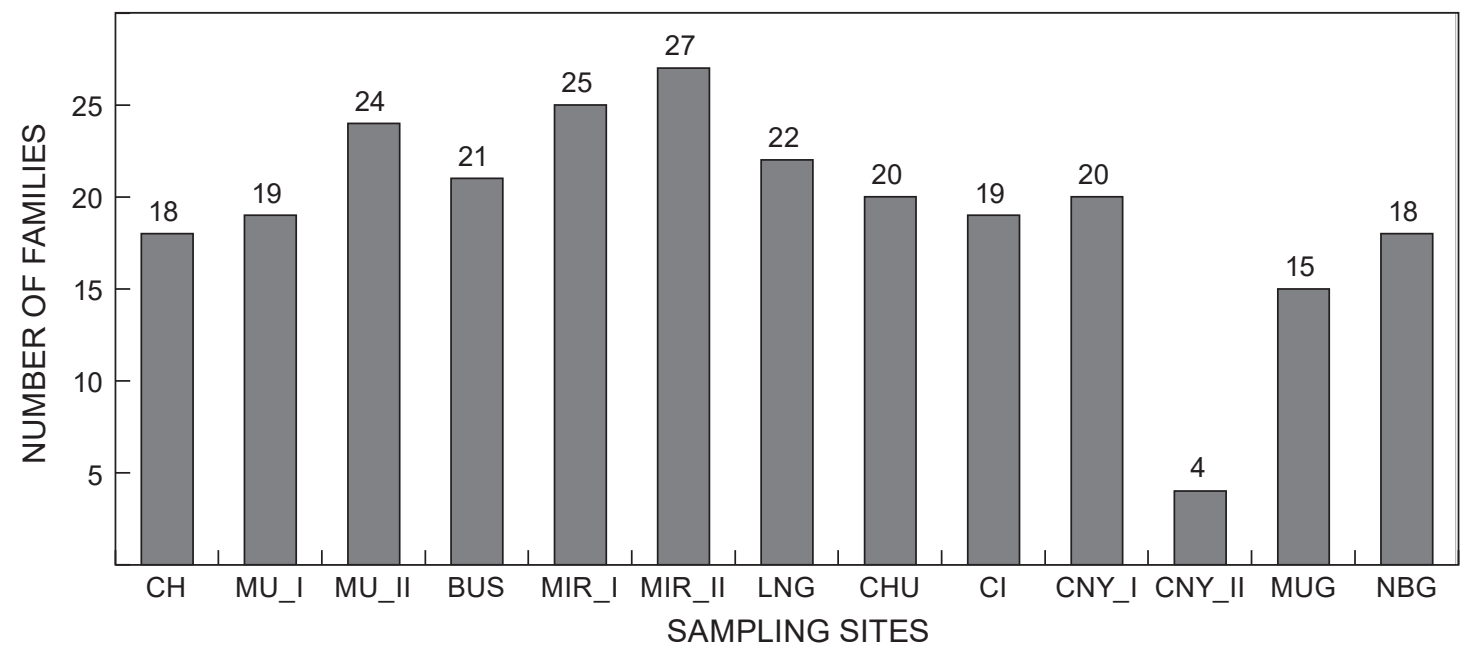

Figure 9: Number of families collected in sampled sites 


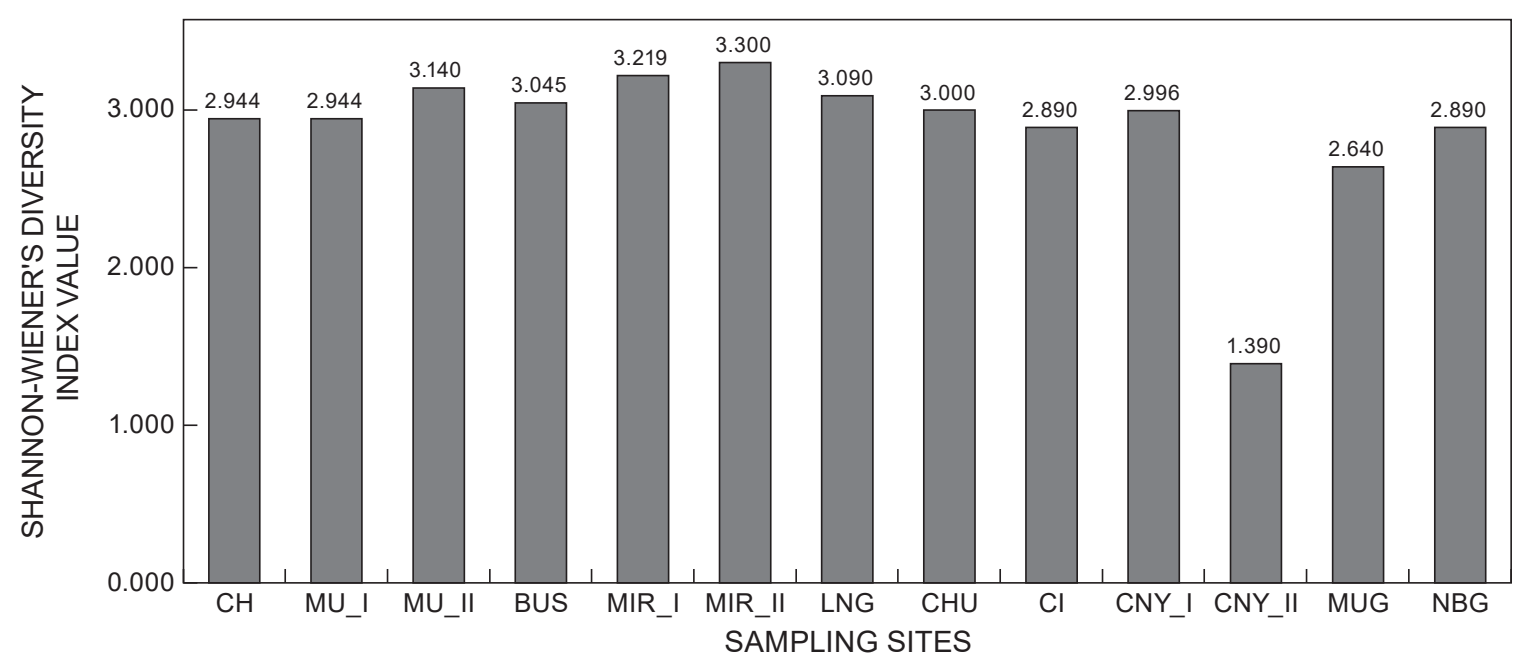

Figure 10: Shannon-Wiener's Diversity Indices for sampled sites

(de Souza et al. 2013; Ogbuagu and Okoli 2013), nutrients uptake (Tabacchi et al. 2000; Dosskey et al. 2010), low total dissolved salts (TDS) (Tabacchi et al. 2000; Wilkinson et al. 2018), low pH (acidic conditions), high water transparency and high dissolved oxygen levels (Collier et al. 1995; WRC 2000; Chapman and Chapman 2003; Kasangaki et al. 2006, 2008; Hussain and Pandit 2012; Wilkinson et al. 2018). The presence of riparian vegetation could therefore explain the low conductivity recorded at sampled sites, although this could also be attributed to geology and soil chemistry, because the KBNP rivers flow over basaltic soils and acidic volcanic rocks (Pecrot and Leonard 1960; Rahm 1967).

Similarly, the acidic conditions observed in the study rivers could be a result of geology and soil chemistry and/ or of the riparian canopy, which provides high levels of organic matter (e.g. decaying plant material that releases humic acid) (Winemiller et al. 2008). In Uganda and the Czech Republic, mountain forest streams showed low conductivity and acidic pH (Kasangaki et al. 2008; Svobodová et al. 2012) as a result of riparian vegetation and forest coverage of watersheds (Dudgeon 2008; Kasangaki et al. 2008; Wilkinson et al. 2018). In addition, the relatively low temperature measured in the current study could be associated with closed-canopy conditions and a high altitude (1 $990 \mathrm{~m}$ to $2384 \mathrm{~m}$ asl), as suggested for Ugandan and South African forest streams (Kasangaki et al. 2006; Dallas 2007a; Dallas 2008). Altitude and canopy cover have indeed previously been reported as fundamental parameters significantly determining water temperature in rivers (Jacobsen 2000; Kasangaki et al. 2006; Dallas 2007a; Rivers-Moore et al. 2008).

Dissolved oxygen levels for most of the sites assessed in the current study were within natural limits to sustain aquatic life (Abowei 2010). However, the low dissolved oxygen concentration observed in CNY_I, CNY_II and MUG were probably related to a low current associated with swamp zones. Dowling and Wiley (1986) reported that a decline in oxygen in slow flowing streams could be attributed to high level of organic matter decay. As a result, slow and stagnant waters combined with the accumulation of decaying plants, increase microbial activity depleting waters from oxygen (Havens et al. 1995). This was particularly striking for CNY_II considering the slow current velocity and aquatic decaying plants, reflecting wetland characteristics of the site. It is a well-know fact that a low dissolved oxygen concentration in aquatic ecosystems could have adverse effects on many aquatic invertebrates when concentrations reach the level of becoming a limiting parameter to sustain life (DWAF 1996; Mason 2002; Dallas and Day 2004; Dallas 2008; Oberholster and Ashton 2008; CSIR 2010; Osibanjo et al. 2010; Nel et al. 2013). Nonetheless, these low dissolved oxygen concentrations and slow current velocity conditions appeared natural in some of the sites suggesting that they are a natural part of the diversity and complexity of KBNP aquatic ecosystems.

The PCA analyses showed that current velocity, conductivity and dissolved oxygen levels were the best drivers of aquatic macroinvertebrates fauna distribution, among the various parameters considered. However, a comparison between the cluster analysis of faunal diversity and the cluster analysis of physico-chemical parameters of the sampling sites revealed that the two clustering patterns differed notably from one (Figure 3 and Figure 8). No direct, strong or obvious relationship between faunal composition of sampled sites and physical and chemical parameters were observed. For example, MU_I and MU_II appeared closely related in terms of physico-chemical parameters (Figure 8), but their macroinvertebrate diversity was completely divergent (Figure 2b). Similarly, CNY-I and CNY_II strongly differed in terms of macroinvertebrate diversity (Figure 2b), yet their physico-chemical parameters were similar (Figure 8). The results suggest that, even if abiotic parameters recorded in this study are known to be crucial ecological factors shaping the aquatic macroinvertebrates community, they were statistically not strong enough to reflect similarities or differences in terms of communities and diversity in the current study. These findings were consistent with the analysis of beta diversity drivers recently conducted by Heino et al. (2015). Additional 
studies are therefore required to determine which abiotic factors are the best indicators of ecological constraints shaping local communities in a particular study area.

\section{Faunal diversity of KBNP Rivers}

The sampling protocol used in the current study was not designed to allow fine quantitative analysis of specimen abundance because the number of collected specimens at each site was strongly dependent on sampling effort and sampled habitat at each site. Despite this, the study proposed one of the very few graphical analysis of relative abundances for the area, providing some interesting insights and new knowledge on the presence and distribution of main taxonomic groups in the relatively un-inventoried rivers of KBNP.

Ephemeroptera and Trichoptera were the most abundant insect orders collected and their presence indicated well-oxygenated water conditions in most investigated rivers. In near natural conditions, small forested streams are often dominated by Trichoptera, Ephemeroptera, Plecoptera and Diptera taxa (Benstead and Pringle 2004). It is worth mentioning that all aquatic macroinvertebrate orders and families reported collected at the sites are widespread in African forest streams (Kasangaki et al. 2006, 2008). The predominance of Ephemeroptera, Trichoptera and Odonata in forest streams has been associated with low temperature, high dissolved oxygen levels, and tolerance of several taxa in these orders towards acidic conditions (Kasangaki et al. 2006, 2008; Lorion and Kennedy 2009; Arthur 2010). Microhabitat diversity is also directly linked to species richness of Ephemeroptera, Trichoptera and Diptera (Silveira et al. 2006; Dallas 2007b) and might be especially important with regards to correlating presence/absence of certain taxa, but the analysis of such component of the habitat was beyond the scope of the current study. Almost all KahuziBiega forest streams share similar features with substrates composed of debris, gravel, pebbles and stones, known to be favourable settlement to benthic macroinvertebrates (See, for example, Beauger and Lair 2008 and references therein).

Although the number of Plecoptera taxa was low, Neoperla (Perlidae) was recorded at most sampling sites. Duran and Suicmez (2007) asserted that high species richness of Ephemeroptera, Plecoptera and Trichoptera indicate good water quality. Unfortunately, current scientific knowledge on ecological traits and requirements of aquatic invertebrates in KBNP is too little to draw any connection between faunal diversity and water quality. Furthermore, the Order Plecoptera is far less diversified in central Africa than in the northern hemisphere. For the studied region, two morphospecies belonging to the same genus were inventoried. Therefore, the relevance of this group for assessing freshwater ecosystems health in central African countries, in comparison with Trichoptera or Ephemeroptera orders, is low. However, because aquatic macroinvertebrates have been successfully used in various countries to assess ecological quality of watercourses (Dallas 1997; Stuijfzand et al. 1999; Dickens and Graham 2002; Houghton 2004; Souto et al. 2011; Pirvu and Pacioglu 2012), it is important to dedicate time and energy to develop a biotic index system to be able to monitor changes in water and ecosystem quality in the
KBNP area. Knowing that Leptophlebiidae (Adenophlebia), Hydropsychidae (Cheumatopsyche, Ethiopsyche) and Perlidae (Neoperla) are sensitive to organic pollution and require clean and well-oxygenated waters to survive in other regions of the world (Tachet et al. 2003; Griffiths et al. 2015), the presence of these taxa at the sampling sites could indicate a general good quality of river ecosystems.

Low diversity at the CNY_II site could be attributed to slow current conditions, associated with muddy substrate, and aquatic decaying plants, leading to low $\mathrm{pH}$ through release of humic and fulvic acids and low dissolved oxygen levels (Dudgeon 2008). The high abundance of chironomid larva (Diptera) and the absence of Trichoptera and Ephemeroptera at the CNY II site were most likely, because of unsuitable substrates and insufficient velocity of the current (hence, unsuitable microhabitats) for numerous species at this site (Móra and Szivák 2012). Nonetheless, those aspects and their influence on the biota and invertebrates diversity were not assessed and, therefore, this interpretation should be considered with care. Some species of chironomid larva (Diptera) are often very tolerant to low dissolved oxygen concentrations, with some species capable of persisting under very low dissolved oxygen levels and acidic conditions, which ultimately might lead to lower diversity (Al-Shami et al. 2010; CSIR 2010; Osibanjo et al. 2010; Souto et al. 2011; Efe et al. 2012; Svobodová et al. 2012; Nel et al. 2013; Ngera 2014). Additional research is nonetheless required to fully understand the reasons for low diversity in some specific sites.

Acentrella (Family Baetidae, Order Ephemeroptera), Plea (Family Pleidae, Order Heteroptera) and Pisidium (Family Sphaeriidae, Order Veneroidea, Phylum Mollusca) were recorded only at the LNG site, which yielded the fourth highest number of morphospecies. The site is located in the forest, close to an agricultural area. During certain periods of the year, farmers used to throw crop waste into rivers in order to clean their fields, resulting elevated decaying vegetation in rivers that cross agricultural lands. These practices may have to nutrient enrichment and subsequent changes in trophic web at LNG. As suggested by the controversial Medium Disturbance Hypothesis (Fox 2013), these periodic disturbances at LNG could lead to a greater richness in species. Kasangaki et al. (2008) previously proposed that at moderately disturbed sites, the disturbance creates ecological space for new species that are not very competitive, consequently increasing the species richness of the site. The study reported the presence of Acentrella genus, which is usually associated with bedrocks and swift flowing and well-oxygenated rivers (Demoulin 1956). Its presence at the LNG site could be explained either by (i) misidentification of these specimens, (ii) tolerance of Acentrella species to extreme conditions in comparison with the ecological optimum that characterises this genus or (iii) the fact that these specimens are species with ecological traits at one extreme of the ecological range of Acentrella genus. Without additional information, the most explanation is the second one. In recent years, studies in rivers flowing through agricultural landscape downstream of the KBNP (Ngera et al. 2009) have revealed about 100 morphospecies, with Baetis (Ephemeroptera), Trichorythus (Ephemeroptera) and Lepidostoma (Trichoptera) as the 
dominant taxa. These taxa are well-known to be more tolerant to organic pollution (Klemm et al. 1990) than those found at the LNG site in the current inventory. It might therefore be interesting to do additional study on the structure of macroinvertebrate species communities and responses to different land-use practices, both inside and outside the protected area of KBNP.

The high morphospecies richness observed in the majority of the sites could be attributed to the diversity of microhabitats (diversity of substratum and current velocity conditions) and undisturbed forested river ecosystems. These results are in line with the conclusions of the programme 'Biodiversité des Écosystèmes Aquatiques et Terrestres dans le Rift Albertin' (P-BEATRA 2005), which reported that the high species richness and diversity recorded in the Kahuzi-Biega Rivers are likely, owing to the natural forested landscape. Several studies showed similar results in other regions across the globe (Klemm et al. 1990; Reece and Richardson 2000; Chapman and Chapman 2003; Dallas 2005; Couceiro et al. 2007; Kasangaki et al. 2008; Lorion and Kennedy 2009). However, the species richness of rivers worldwide usually peaks for mid-order streams and large rivers, as opposed to headwater rivers, because of a greater diversity of habitats and flow conditions in comparison with small streams and rivers (Ward and Stanford 1983; Hussain and Pandit 2012).

\section{Conclusion}

This study, and previous inventories in KBNP (P-BEATRA 2005), improved the knowledge of aquatic macroinvertebrates biodiversity in this area of the Albertine Rift (central Africa). Although some species have not been described, aquatic macroinvertebrates collected in the current study were not restricted to Kahuzi-Biega rivers and streams, and all appear to be widespread in forest rivers in the region. Three decades ago, studies in the vicinity of KBNP revealed 21 and 26 species belonging to Ephemeroptera and Trichoptera respectively (Statzner 1976; Kopelke 1979, 1981). This information emphasises the requirement for knowledge on the taxonomic diversity of aquatic macroinvertebrates in KBNP region to be synthesised. One can imagine, for example, the construction of an online database of the aquatic species of the region, coupled with geographical data for a better understanding of the spatial ecology of these species. These are needed to ground future ecological work as shown in this study.

More in-depth taxonomic (species-level identification) and ecological studies would lead to a better understanding of these taxa and their suitability as indicators for different ecological conditions. Extending the sampling effort all year round, including the lowland region of the park, would also facilitate understanding of ecological processes in KahuziBiega rivers and streams. More comprehensive sampling protocols of biota would have to be developed, including light and Malaise trapping methods, because some species rely on adult stages for species identification. This would provide valuable information on the ecological status of KBNP rivers and help conservationists to plan and implement conservation strategies. Indeed, KBNP both an area of major ecological interest and one of extreme socioeconomic importance. It is therefore crucial to be able to conserve and manage this protected area in a sustainable way, based on reliable scientific knowledge.

Acknowledgements - We thank the Field Museum of Chicago through John Bates for financial support of this field work, the KBNP Director (Radar Nashuli) and CRSN authorities that allowed us access to the park. Our gratitude goes also to Helen Barber-James (Albany Museum, Department of Freshwater Invertebrates, Grahamstown, South Africa) for her assistance with macroinvertebrates identification and Kitambala wa Kayugi for his help in the field. We also express our sincerest gratitude to Julian Kerbis for his advice and encouragement and to Betty Chang and Anneloes Martinsen for reviewing the English of the manuscript. We also express our gratitude to the anonymous referees that reviewed our manuscript and made valuable comments that helped us to improve the manuscript.

\section{References}

Abel PD. 2002. Water Pollution Biology. (2nd Ed.). Taylor and Francis Ltd.

Abowei JFN. 2010. Salinity, Dissolved oxygen, pH and Surface water Temperature conditions in Nkoro River, Niger Delta, Nigeria. Advance Journal of Food Science and Technology 2(1): 36-40.

Al-Shami SA, Rawi CSM, Hassan Ahmad A, Nor SAM. 2010. Distribution of Chironomidae (Insecta: Diptera) in polluted rivers of the Juru River Basin, Penang, Malay. Journal of Environmental Sciences (China) 22(11): 1718-1727.

Arthur JB. 2010. The influence of upstream forest on macroinvertebrate communities in pastoral landscapes. MSc thesis, University of Canterbury, Great Britain.

Bagalwa M, Zirirane N, Pauls S, Karume K, Ngera M, Bisimwa M, Mushagalusa NG. 2012. Aspects of the physic-chemical characteristics of rivers in Kahuzi-Biega National Park, Democratic Republic of Congo. Journal of Environmental Protection 3: 1590-1595.

Barnaud G, Fustec E. 2007. Conserver les zones humides: pourqoi? Comment? Dijon, France: Éditions Quae, Versailles, and Educagri éditions.

Beauger A, Lair N. 2008. Keeping it simple: benefits of targeting riffle-pool macroinvertabrate communities over multi-substratum sampling protocols in the preparation of a new European biotic index. Ecological Indicators 8: 555-563.

Benstead JP, Pringle CM. 2004. Deforestation alters the resource base and biomass of endemic stream insects in eastern Madagascar. Freshwater Biology 49: 490-501.

Bisimwa MA, Ngera MF, Bisimwa KD, Bagalwa MJ, Mushayuma NH. 2013. A Preliminary Checklist of Epilithic Algae of Kahuzi-Biega National Park, Democratic Republic of the Congo. Greener Journal of Biological Science 3(8): 282-291.

Chapman CA, Chapman LJ. 2003. Deforestation in Tropical Africa. Impacts on aquatic ecosystems. Gainesville: University Press of Florida.

Chutter FM. 1972. An empirical biotic index of the quality of water in South African Streams and Rivers. Water Research Pergamon 6: 19-30.

Collier KJ, Cooper AB, Davies-Colley RJ, Rutherford JC, Smith CM, Williamson RB. 1995. Managing riparian zones: A contribution to protecting New Zealand's rivers and streams. Vol 1: Concepts. Wellington, New Zealand: Department of Conservation.

Couceiro SRM, Hamada N, Luz SLB, Forsberg BR, Pimentel TP. 2007. Deforestation and sewage effects on aquatic macroinvertebrates in urban streams in Manaus, Amazonas, Brazil. Hydrobiologia 575(1): 271-284. 
CSIR 2010. A CSIR Perspective on Water in South Africa. Report No. CSIR/NRE/PW/IR/2011/0012/A. ISBN: 978-0-7988-55952. Pretoria, South Africa: Council for Scientific and Industrial Research.

Dallas HF. 2008. Water temperature and riverine ecosystems: An overview of knowledge and approaches for assessing biotic responses, with special reference to South Africa. Water SA 34(3): 393-404.

Dallas HF 2007a. The influence of biotope availability on macroinvertebrate assemblages in South African rivers: implications for aquatic bioassessment. Freshwater Biology 52: 370-380.

Dallas HF 2007b. The effect of biotope-specific sampling for aquatic macroinvertebrates on reference site classification and the identification of environmental predictors in Mpumalanga, South Africa. African Journal of Aquatic Science 32(2): 165-173.

Dallas HF. 2005. River health programme: site characterisation field-manual and field-data sheets. Pretoria, South Africa: Resource Quality Services, Department of Water Affairs and Forestry.

Dallas HF 1997. A preliminary evaluation of aspects of SASS (South African Scoring System) for the rapid bioassessment of water quality in rivers, with particular reference to the incorporation of SASS in a national biomonitoring programme. Southern African Journal of Aquatic Sciences 23(1): 79-94.

Dallas HF, Day JA. 2004. The Effect of Water Quality Variables on Aquatic Ecosystems. 222 pp. WRC Report No 224/04. Gezina, South Africa: Water Research Commission.

Davies PJ, Wright IA, Findlay SJ, Jonasson OJ, Burgin S. 2010. Impact of urban development on aquatic macroinvertebrates in South Eastern Australia: degradation of in-stream habitats and comparison with non-urban. Aquatic Ecology 44: 685-700.

Davies B. Day J. 1998. Vanishing Waters. 487 pp. Cape Town: University of Cape Town Press.

De Moor IJ, Day JA, de Moor FC. 2003a. Guides to the Freshwater Invertebrates of Southern Africa. Volume 8: Insect II. Hemiptera Megaloptera, Neuroptera, Trichoptera and Lepidoptera. WRC Report No TT 214/03. Gezina, South Africa: Water Research Commission.

De Moor IJ, Day JA, de Moor FC. 2003b. Guides to the Freshwater Invertebrates of Southern Africa. Volume 7: Insect I. Ephemeroptera, Odonata \& Plecoptera. WRC Report No TT 207/03. Gezina, South Africa: Water Research Commission.

De Souza A., Fonseca D, Libório R, Tanaka M. 2013. Influence of riparian vegetation and forest structure on the water quality of rural low-order streams in SE Brazil. Forest Ecology and Management 298: 12-18.

Demoulin G. 1956. Ephemeroptera. 3 (7) in Résultats scientifiques de l'exploration hydrobiologiques du lac Tanganika (1946-1947). Belgium: Institut Royal des Sciences Naturelles de Belgique.

Dickens CWS, Graham, PM. 2002. The South African Scoring System (SASS) version 5 rapid bioassessment method for rivers. African Journal of Aquatic Science 27: 1-10.

Dosskey M, Vidon P, Gurwick N, Allan C, Duval T, Lowrance R. 2010. The role of riparian vegetation in protecting and improving chemical water quality in streams. Journal of the American Water Resources Association (JAWRA): 1-18. Paper No. JAWRA-09-0035-P.

Dowling DC, Wiley MJ. 1986. The effects of dissolved oxygen, temperature, and low stream flow on fishes: A literature review. Urbana-Champaign, USA: University of Illinois.

Dugeon D (Ed.). 2008. Tropical stream ecology. 343 pp. The Netherlands, Amsterdam: Elsevier Academic Press.

Duran M, Suicmez M. 2007. Utilization of both benthic macroinvertebrates and physicochemical parameters for evaluating water quality of the stream Cekerek (Tokat, Turkey). Journal of Environmental Biology 28(2): 231-236.

Duran M. 2006. Monitoring water quality using benthic macroinvertebrates and physicochemical parameters of Behzat Stream in Turkey. Polish Journal of Environmental Studies 15(5): 709-719.
DWAF 1996. South African Water Quality Guidelines. Vol 7. Aquatic Ecosystems. Pretoria, South Africa: Department of Water Affairs and Forestry.

Fox J. 2013. The intermediate disturbance hypothesis should be abandoned. Trends in Ecology and Evolution 28(2): 86-92.

Griffiths CH, Day J, Picker M (Eds). 2015. Freshwater Life. A field guide to the plants and animals of southern Africa. 368 pp. Cape Town, South Africa. Struik Nature/Penguin Random House Press.

Guerold F. 2000. Influence of taxonomic determination level on several community indices. Water Research 34(2): 487-492.

Havens KJ, Varnell LM, Bradshaw JG. 1995. An assessment of ecological conditions in a constructed tidal marsh and two natural reference tidal marshes in coastal Virginia. Ecological Engineering 4: 117-141.

Heino J, Melo A, Mauricio Bini L, Altermatt F, Al-Shami S, Angeler D, et al. A comparative analysis reveals weak relationships between ecological factors and beta diversity of stream insect metacommunities at two spatial levels. Ecology and Evolution 5(6): 1235-1248.

Houghton D. 2004. Utility of Caddisflies (Insecta: Trichoptera) as Indicators of Habitat Disturbance in Minnesota. Journal of Freshwater Ecology 19(1): 97-108.

Hussain AQ, Pandit KA. 2012. Macroinvertebrates in streams: A review of some ecological factors. International Journal of Fisheries and Aquaculture 4(7): 114-123.

Jacobsen D. 2000. Gill size of trichopteran larvae and oxygen supply in streams along a 4000-m gradient of altitude. Journal of the North American Benthological Society 19(2): 329-343.

Jaquemart S, Statzner B. 1981. Trichoptères nouveaux du Zaïre. Bulletin de l'Institut Royal des Sciences Naturelles de Belgique. 53(21): 1-25.

Kasangaki A, Babaasa D, Efitre J, McNeilage A, Bitariho R. 2006. Links between anthropogenic perturbations and benthic macroinvertebrate assemblages in Afromontane forest streams in Uganda. Hydrobiologia 563: 231-245.

Kasangaki A, Chapman LJ, Balirwa J. 2008. Land use and the ecology of benthic macroinvertebrate assemblages of high-altitude rainforest streams in Uganda. Freshwater Biology 53(4): 681-697.

Kasereka B. 2003. Factors Affecting the Boundary Demarcation in the Kahuzi-Biega National Park, Kivu, D. R. Congo. African Study Monographs 24(3): 181-194.

Klemm DJ, Lewis PA, Fulk F, Lazorchak JM. 1990. Macroinvertebrate field and laboratory methods for evaluating the biological integrity of surface waters. EPA/600/4-90/030. Cincinnati, Ohio, United States of America: United States Environmental Protection Agency.

Kopelke JP. 1979. Ephemeroptera aus der Emergenz des Zentralafrikanischen Bergbaches Kalengo (Zaire). Teil I Baetidae. Entomologische Abhandlungen Museum für Tierkunde Dresden 43: 233-239.

Kopelke JP. 1981. Ephemeroptera (Insecta) aus der Emergenz des Zentralafrikanischen Bergbaches Kalengo. II. Leptophlebiidae, Heptageniidae, Trichorythidae, Caenidae. Bulletin de la société entomologique suisse 54: 139-156.

Liengola BI. 2008. Impact of the Invasive Liana Sericostachys scandens on Forest Composition: Implications for the Recovery of Grauer's Gorilla in the Kahuzi-Biega National Park, Democratic Republic of Congo. The Bulletin of the Yale Tropical Resource Institute 27: 43-50.

Lorion CM, Kennedy BP. 2009. Relationships between deforestation, riparian forest buffers and benthic macroinvertebrates in Neotropical headwater streams. Freshwater Biology 54: 165-180.

Mackie G. 2004. Applied aquatic ecosystem concepts. 757 pp. lowa: Kendall/Hunt Publishing Company.

Marlier G. 1954a. Ecologie et éthologie des Trichoptères du Congo oriental. Annales du Museum Royal du Congo belge, Sciences Zoologiques, Tervuren, Belgique 1: 457-463.

Marlier G. 1954b. Recherches hydrobiologiques dans les rivières du Congo Oriental. II. Hydrobiologia 6: 225-264. 
Marlier G. 1961. Hydropsychidae du Kivu (Trichoptera). Revue de Zoologie et de Botanique Africaine 63: 158-212.

Marlier G. 1962. Genera des Trichopteres de l'Afrique. Tervuren, Belgique 109: Annales du Musée Royal de l'Afrique Centrale.

Mason C. 2002. Biology of Freshwater Pollution. 387 pp. 4th Edition. Harlow, England: Pearson/Prentice Hall Education.

Móra A, Szivák I. 2012. Spatial distribution and diversity of chironomid assemblages in a small hilly stream (Diptera: Chironomidae). Aquatic Insects 341: 127-138.

Mühlenberg M, Slowik J, Steinhauer-Burkart B. 1995. Kahuzi-Biéga National Park. Kinshasa, Zaïre: IZCN/GTZ

Mutimanwa DK. 2001. The Bambuti-Batwa and the Kahuzi-Biega National Park: The Case of the Barhwa and Babuluko People of the PNKB. in: Indigenous People and Protected Areas in Africa: Forest People Project Report: 87-110. Moreton-in-Marsh, England: Forest peoples programme.

Nel N, Parker A, Silbernagl P. 2013. Improving water quality in stormwater and river systems: an approach for determining resources. Journal of the South African Institution of Civil Engineering 55(1): 22-35.

Ngera MF, Cammaerts D, Bisimwa M, Baluku B. 2009. Etude comparative de macroinvertebres benthiques de trois cours d'eau du bassin versant du Lac Kivu en DR Congo. Cahier du CERUKI, Numéro special CRSN-Lwiro: 89-94. Bukavu, DRCongo: Institut Supérieur Pédagogique de Bukavu.

Ngera MF. 2014. Spatial variation in selected water quality parameters in the upper Kuils River (Western Cape Province, South Africa). MSc thesis. University of the Western Cape, South Africa.

Oberholster PJ, Ashton PJ. 2008. An overview of the current status of water quality and eutrophication in South African Rivers and Reservoirs. State of the Nation Report. Pretoria, South Africa: Council for Scientific and Industrial Research.

Ogbuagu D, Okol C. 2013. What influence does removal of riparian vegetation have on primary productivity of a river? Central European Journal of Experimental Biology 2(2): 5-12.

Ogidiaka E, Esenowo IK, Ugwumba AA. 2012. Physico-Chemical Parameters and Benthic Macroinvertebrates of Ogunpa River at Bodija, Ibadan, Oyo State. European Journal of Scientific Research 85(1): 89-97.

Osibanjo O, Daso AP, Gbadebo AM. 2010. The impact of industries on surface water quality of River Ona and River Alaro in Oluyole Industrial Estate, Ibadan, Nigeria. African Journal of Biotechnology 10(4): 696-702.

Pauls SU, Holzenthal RW, Ngera MF. 2010. Two new species of Triaenodes MCLACHLAN 1865 from streams in the Lake Kivu basin, South Kivu, Democratic Republic of the Congo (Trichoptera, Leptoceridae). Denisia 29: 277-285.

P-BEATRA. 2005. Etude de la Biodiversité des Ecosystèmes du Parc National de Kahuzi-Biega et du Lac Tanganyika et ses affluents à Uvira, Est de la République Démocratique du Congo. Rapport de la Session de Formation de Juillet-Août 2005. Chicago, United States of America: John D and Catherine T MacArthur Foundation.

Pecrot A, Leonard A. 1960. Note Explicative de la Carte des Sols et de la Végétation du Congo Belge et du Rwanda-Urundi. Léopoldville, Congo Belge: Institut National pour l'Etude Agronomique du Congo Belge (INEAC).

Pirvu M, Pacioglu O. 2012. The ecological requirements of caddisflies larvae (Insecta: Trichoptera) and their usefulness in water quality assessment of a river in south-west Romania. Knowledge and Management of Aquatic Ecosystems 407(3).

Poole RW. 1974. An introduction to quantitative ecology. New York: McGraw-Hill.

Rahm U. 1967. Les muridés des environs du Lac Kivu et des régions voisines (Afrique Centrale) et leur ecologie. Revue Suisse de Zoologie 74(9): 439-520.
Rivers-Moore NA, Hughes DA, Mantel S. 2008. Links between water temperatures, ecological responses and flow rates: a framework for establishing water temperature guidelines for the ecological reserve. Water Research Commission Report No KV 214/08. 67 pp. Pretoria, South Africa: Water Research Commission.

Reece PF, Richardson JS. 2000. Benthic macroinvertebrate assemblages of coastal and continental streams and large rivers of southwestern British Columbia, Canada. Hydrobiologia 439: 77-89.

Rosenberg DM, Resh VH. 1993. Introduction to freshwater biomonitoring and benthic macroinvertebrates, pp. 1-9. In: Rosenberg DM, Resh VH (Eds) Freshwater biomonitoring and benthic macroinvertebrates. New York: Chapman and Hall.

Silveira MP, Buss DF, Nessimian JL, Baptista DF. 2006. Spatial and temporal distribution of benthic macroinvertebrates in a South Eastern Brazilian River. Brazilian Journal of Biology 66(2B): 623-632.

Souto RMG, Facure KG, Pavanin LA, Jacobucci GB. 2011. Influence of environmental factors on benthic macroinvertebrate communities on urban streams in Vereda habitats, Central Brazil. Acta Limnologica Brasiliensia 23(3): 293-306.

Spellerberg IF, Fedor PJ. 2003. A tribute to Claude Shannon (1901-2001) and a plea for more rigorous use of species richness, species diversity and the Shannon-Wiener Index. Global Ecology and Biogeography 12: 177-179.

Statzner B. 1975. Longitudinal zonation of a Central African running water system with particular reference to caddisflies (Trichoptera, Insecta). Archiv für Hydrobiologie 76: 153-180.

Statzner B. 1976. The emergence of caddis flies (Trichoptera, Insecta) from the Central African mountain stream Kalengo. Archiv für Hydrobiologie 78: 102-137.

Stuijfzand SC, Engels S, van Ammelrooy E, Jonker M. 1999. Caddisflies (Trichoptera: Hydropsychidae) Used for Evaluating Water Quality of Large European Rivers. Archives of Environmental Contamination and Toxicology 36: 186-192.

Sundermann A, Lohse SLA, Beck LA, Haase P. 2007. Key to the larval stages of aquatic true flies (Diptera), based on the operational taxa list for running waters in Germany. International Journal of Limnology 43(1): 61-74.

Svobodová J, Matena J, Kopacek J, Polakova S, Vrba J. 2012. Spatial and temporal changes of benthic macroinvertebrate assemblages in acidified streams in the Bohemian Forest (Czech Republic). Aquatic Insects 34 Supplement 1: 157-172.

Tabacchi E, Lambs L, Guilloy H, Planty-Tabacchi A-M, Muller E, Décamps H. 2000. Impacts of vegetation on hydrological processes. Hydrological Processes 14(16-17): 2959-2976.

Tachet H, Bournaud M, Usseglio-Polatera P. 2003. Invertébrés d'eau douce. Systématique, Biologie, Ecologie. 587 pp. Paris, France: Centre nationale pour la Recherche Scientifique Editions.

UNEP-WCMC. 2007. Kahuzi-Biega National Park Democratic Republic of the Congo. International Union for the Conservation of Nature and United Nations Environmental Program. World Conservation Monitoring Centre. New-York,New-York, United States of America: United Nations Environmental Program-World Conservation Monitoring Centre.

UNEP-WCMC. 2009. "Kahuzi-Biéga National Park, Democratic Republic of Congo". In: Encyclopedia of Earth. Cutler J (Ed.). Cleveland (Washington, DC: Environmental Information Coalition, National Council for Science and the Environment). New-York, United States of America: United Nations Environmental Program-World Conservation Monitoring Centre.

Ward J, Stanford J. 1983. The serial discontinuity concept of lotic ecosystems. In: Fontaine T, Bartell S (Eds). Dynamics of lotic ecosystems. pp. 29-42. Michigan, United States of America: Ann Arbor Science.

Wilkinson H, Spiller B, Forbes N, Ortega-Achury S, RamirezAvila J. 2018. The effects of forested riparian zones on stream 
conditions. Dallas, United States of America: American Society of Civil Engineers (ASCE)

Winemiller K, Agostinho A, Caramaschi E. 2008. Fish ecology in tropical streams. pp 107-140. In: Dugeon D (Ed.) Tropical stream ecology. The Netherlands: Amsterdam, Elsevier Academic Press.

WRC. 2000. Water notes. The values of riparian zone. Water and
Rivers Commission (WRC) River Restoration Report No. RR 2. East Perth, Australia: Water and Rivers Commission (WRC).

Ziglio G, Siligardi M, Flaim G (Eds). 2006. Biological Monitoring of Rivers. Applications and perspectives. Chichester, England: John Wiley \& Sons, Ltd.

Zoltai S, Vitt D. 1995. Canadian wetlands: Environmental gradients and classification. Vegetatio 118: 131-137. 